\title{
Predicting NMR Spectra by Computational Methods: Structure Revision of Hexacyclinol
}

\author{
Scott D. Rychnovsky \\ Department of Chemistry, 1102 Natural Sciences II, \\ University of California, Irvine, Irvine, California, 92697-2025
}

\section{Table of Contents}

Calculated structures and ${ }^{13} \mathrm{C}$ NMR chemical shifts:

Hexacyclinol (1)

Elisapterosin B

Maoecrystal V

Elisabethin A

Hexacyclinol 7 conformation 1

Hexacyclinol 7 conformation 2

Stereoisomer 6-D

Stereoisomer 6-B

Stereoisomer 6-A
Page S2

Page S5

Page S8

Page S11

Page S14

Page S19

Page S23

Page S27

Page S31

Gaussian 03: Revision C.02, M. J. Frisch, G. W. Trucks, H. B. Schlegel, G. E. Scuseria, M. A. Robb, J. R. Cheeseman, J. A. Montgomery, Jr., T. Vreven, K. N. Kudin, J. C. Burant, J. M. Millam, S. S. Iyengar, J. Tomasi, V. Barone, B. Mennucci, M. Cossi, G. Scalmani, N. Rega, G. A. Petersson, H. Nakatsuji, M. Hada, M. Ehara, K. Toyota, R. Fukuda, J. Hasegawa, M. Ishida, T. Nakajima, Y. Honda, O. Kitao, H. Nakai, M. Klene, X. Li, J. E. Knox, H. P. Hratchian, J. B. Cross, C. Adamo, J. Jaramillo, R. Gomperts, R. E. Stratmann, O. Yazyev, A. J. Austin, R. Cammi, C. Pomelli, J. W. Ochterski, P. Y. Ayala, K. Morokuma, G. A. Voth, P. Salvador, J. J. Dannenberg, V. G. Zakrzewski, S. Dapprich, A. D. Daniels, M. C. Strain, O. Farkas, D. K. Malick, A. D. Rabuck, K. Raghavachari, J. B. Foresman, J. V. Ortiz, Q. Cui, A. G. Baboul, S. Clifford, J. Cioslowski, B. B. Stefanov, G. Liu, A. Liashenko, P. Piskorz, I. Komaromi, R. L. Martin, D. J. Fox, T. Keith, M. A. Al-Laham, C. Y. Peng, A. Nanayakkara, M. Challacombe, P. M. W. Gill, B. Johnson, W. Chen, M. W. Wong, C. Gonzalez, and J. A. Pople, Gaussian, Inc., Wallingford CT, 2004. 
Hexacyclinol structure 1 optimized at $\mathrm{HF} / 3-21 \mathrm{G}$

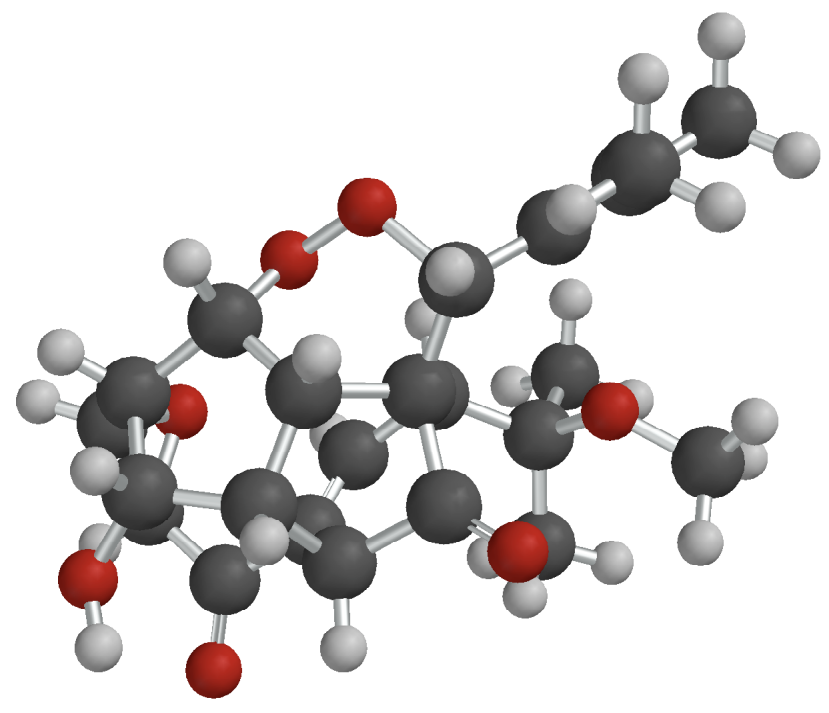

$\begin{array}{llll}\mathrm{C} & -0.0631693951 & 0.8216082563 & -1.5374819285 \\ \mathrm{C} & -0.0615161666 & 0.820389927 & -0.0044957383 \\ \mathrm{C} & 1.5284571417 & 0.8213909172 & 0.1191088184 \\ \mathrm{C} & 2.1689663037 & 0.2555867909 & -1.1566202394 \\ \mathrm{C} & 0.9372220175 & -0.3665134828 & -1.8394419471 \\ \mathrm{H} & 1.7889087229 & 1.8652719128 & 0.197006923 \\ \mathrm{H} & 2.560319039 & 1.0514153651 & -1.7803450072 \\ \mathrm{H} & 1.0337782342 & -0.5444539928 & -2.9001125512 \\ \mathrm{O} & -0.5491691462 & 1.5385990201 & -2.3691260779 \\ \mathrm{C} & 2.0499669502 & 0.0890011937 & 1.3423173673 \\ \mathrm{H} & 2.7019717939 & 0.7385874235 & 1.9050406436 \\ \mathrm{C} & 3.3409912811 & -0.590112039 & -0.6118932177 \\ \mathrm{H} & 4.1540632928 & 0.1166686444 & -0.4766537459 \\ \mathrm{C} & 2.9671484687 & -1.0936905131 & 0.8271449361 \\ \mathrm{H} & 3.8750173677 & -0.9980571246 & 1.4076560421 \\ \mathrm{O} & 0.8824538249 & -0.1272425607 & 2.1751559165 \\ \mathrm{O} & 3.7965709363 & -1.6899038181 & -1.3986665998 \\ \mathrm{H} & 3.9086363347 & -1.4567150391 & -2.3292273229 \\ \mathrm{C} & 0.6275399289 & -1.6361510709 & -1.0760257559 \\ \mathrm{C} & -0.2461682747 & -1.6696871349 & -0.0962919775 \\ \mathrm{H} & -0.4246264036 & -2.557511005 & 0.4625945557 \\ \mathrm{C} & -0.9663233341 & -0.4405618034 & 0.2952818205 \\ \mathrm{C} & 1.4433329876 & -2.8327509164 & -1.3588078881 \\ \mathrm{O} & 1.6572059015 & -3.2785329046 & -2.4653676166 \\ \mathrm{C} & 2.0754604289 & -3.4551883844 & -0.1505629752 \\ \mathrm{H} & 2.4788056864 & -4.4320278823 & -0.3165017964 \\ \mathrm{C} & 2.7093448754 & -2.6061539069 & 0.8855883717 \\ \mathrm{H} & 3.4972912461 & -3.1049147709 & 1.413353946 \\ \mathrm{O} & 1.4319488967 & -3.2568087715 & 1.1567205613\end{array}$




$\begin{array}{llll}\mathrm{C} & -2.3944471 & -0.4007348563 & -0.3924313557 \\ \mathrm{C} & -2.4889709418 & -1.1508924212 & -1.7355417457 \\ \mathrm{H} & -1.8385967744 & -0.7369492235 & -2.4901724584 \\ \mathrm{H} & -3.5064204975 & -1.0975639787 & -2.1033216512 \\ \mathrm{H} & -2.2425535231 & -2.1932459129 & -1.6012701838 \\ \mathrm{C} & -3.407739458 & -1.0362430969 & 0.5760804762 \\ \mathrm{H} & -4.4022764909 & -1.0241674074 & 0.1449476974 \\ \mathrm{H} & -3.4333983511 & -0.4859287949 & 1.5063739702 \\ \mathrm{H} & -3.1410467219 & -2.0659702567 & 0.7838302886 \\ \mathrm{O} & -2.6885440393 & 0.9986733716 & -0.5323964355 \\ \mathrm{C} & -3.6654346008 & 1.4665359517 & -1.4723992772 \\ \mathrm{H} & -4.6205024145 & 0.9678273979 & -1.3537823467 \\ \mathrm{H} & -3.3175442076 & 1.3530801952 & -2.4881952643 \\ \mathrm{H} & -3.7885275676 & 2.5169152763 & -1.2577074204 \\ \mathrm{C} & -0.3450628743 & 1.8683726842 & 1.091075391 \\ \mathrm{H} & 0.2369415658 & 2.7500010795 & 0.9021770889 \\ \mathrm{O} & 0.3373100832 & 1.246716405 & 2.342255415 \\ \mathrm{H} & -1.1301877348 & -0.4687194136 & 1.3557985285 \\ \mathrm{C} & -1.6962086773 & 2.2405242069 & 1.5946462663 \\ \mathrm{H} & -2.3021995961 & 1.4255450286 & 1.9195146081 \\ \mathrm{C} & -2.1505717542 & 3.4713587864 & 1.7465762903 \\ \mathrm{C} & -1.4440536741 & 4.7534869619 & 1.3530772312 \\ \mathrm{H} & -0.457068898 & 4.600278515 & 0.9430786491 \\ \mathrm{H} & -1.3538531724 & 5.4033841786 & 2.2186127806 \\ \mathrm{H} & -2.034205009 & 5.2803422426 & 0.6087321898 \\ \mathrm{C} & -3.5179888297 & 3.7166387062 & 2.3562134467 \\ \mathrm{H} & -4.1537408065 & 4.2519540899 & 1.6564755705 \\ \mathrm{H} & -3.4276572568 & 4.3267165567 & 3.2505956891 \\ \mathrm{H} & -4.0081913334 & 2.7889424237 & 2.6222010572\end{array}$

$H F=-1403.1603757$ 


\begin{tabular}{|c|c|c|c|c|c|}
\hline $\begin{array}{l}\text { Carbon } \\
\text { Number }\end{array}$ & $\begin{array}{l}\text { Chemical } \\
\text { shift }\end{array}$ & $\begin{array}{l}\text { mPW1PW91/ } \\
6-31 G(d, p)\end{array}$ & Corrected & difference & |difference| \\
\hline 1 & 18.6 & 17.6 & 12.1 & -6.5 & 6.5 \\
\hline 2 & 142.2 & 128.1 & 129.6 & -12.6 & 12.6 \\
\hline 3 & 26.1 & 26.7 & 21.7 & -4.4 & 4.4 \\
\hline 4 & 120.7 & 122.4 & 123.5 & 2.8 & 2.8 \\
\hline 5 & 75.8 & 98.2 & 97.8 & 22.0 & 22.0 \\
\hline 6 & 60.5 & 59.3 & 56.4 & -4.1 & 4.1 \\
\hline 7 & 202.9 & 193.1 & 198.7 & -4.2 & 4.2 \\
\hline 8 & 53.1 & 57.1 & 54.1 & 1.0 & 1.0 \\
\hline 9 & 54.5 & 41.9 & 37.9 & -16.6 & 16.6 \\
\hline 10 & 47.8 & 58.1 & 55.1 & 7.3 & 7.3 \\
\hline 11 & 71.5 & 90.3 & 89.4 & 17.9 & 17.9 \\
\hline 12 & 40.4 & 48.2 & 44.6 & 4.2 & 4.2 \\
\hline 13 & 72.7 & 77.3 & 75.6 & 2.9 & 2.9 \\
\hline 14 & 61 & 57.1 & 54.1 & -6.9 & 6.9 \\
\hline 15 & 53.2 & 59.6 & 56.7 & 3.5 & 3.5 \\
\hline 16 & 192.8 & 194.3 & 200.0 & 7.2 & 7.2 \\
\hline 17 & 132.5 & 125.9 & 127.3 & -5.2 & 5.2 \\
\hline 18 & 139.6 & 138.1 & 140.2 & 0.6 & 0.6 \\
\hline 19 & 40.9 & 57.4 & 54.4 & 13.5 & 13.5 \\
\hline 20 & 77.3 & 79.3 & 77.7 & 0.4 & 0.4 \\
\hline 21 & 26.6 & 28.4 & 23.5 & -3.1 & 3.1 \\
\hline 22 & 24.7 & 23.4 & 18.2 & -6.5 & 6.5 \\
\hline 23 & 49.1 & 49.2 & 45.7 & -3.4 & 3.4 \\
\hline & & & & $\begin{array}{l}\text { average } \\
\max \end{array}$ & $\begin{array}{r}6.8 \\
22.0\end{array}$ \\
\hline
\end{tabular}

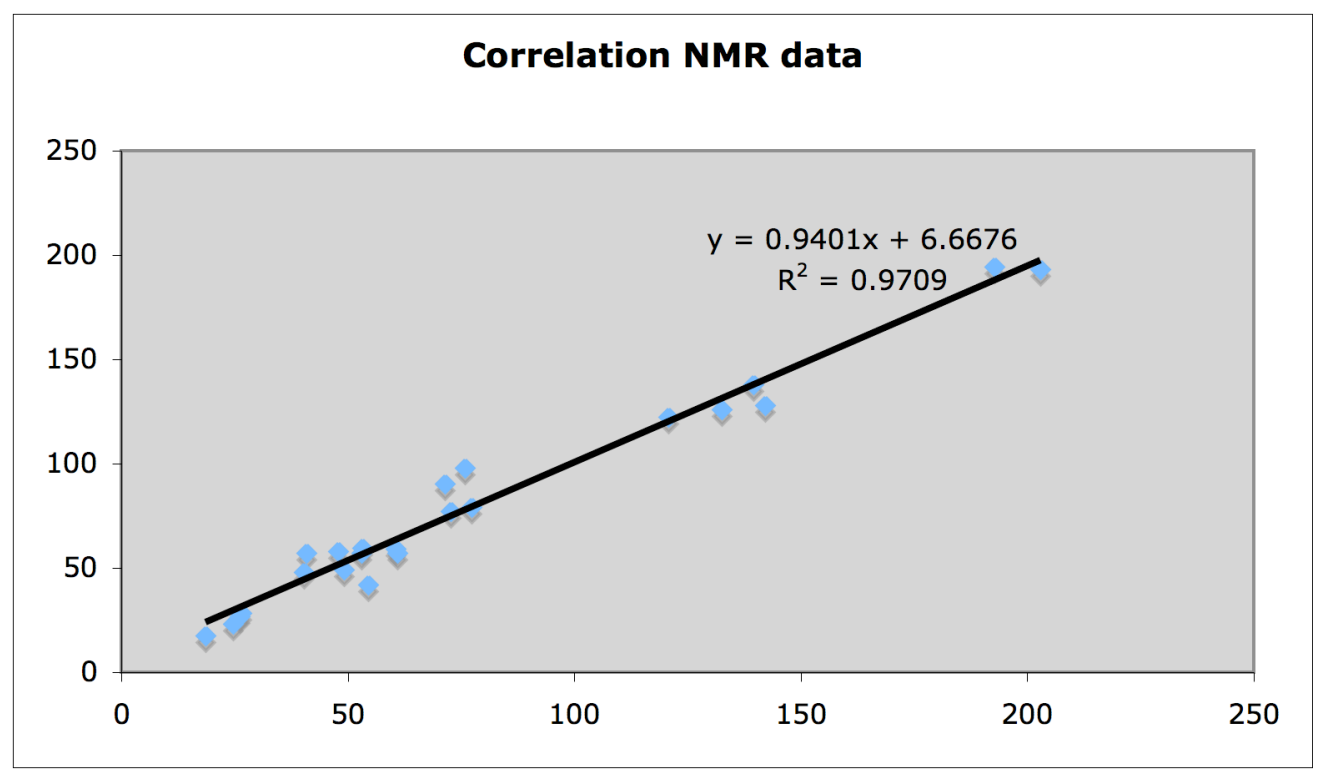


Elisapterosin B structure optimized at $\mathrm{HF} / 3-21 \mathrm{G}$

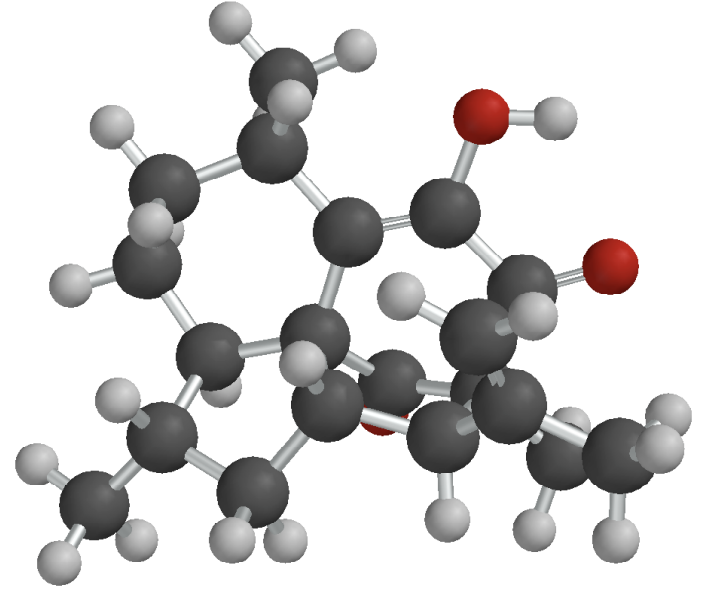

$\begin{array}{llll}\mathrm{H} & -2.554864447 & 0.4558923749 & -1.9856384883 \\ \mathrm{C} & -2.5536353944 & 0.4589837175 & -0.9011198806 \\ \mathrm{C} & -0.3641334953 & 0.4607613092 & 0.5765789632 \\ \mathrm{C} & -2.3998804854 & 1.9817130959 & 1.1179495803 \\ \mathrm{C} & -0.9594027013 & 1.5748202216 & 1.4771936178 \\ \mathrm{C} & -2.6394350637 & 1.9103588423 & -0.3966707749 \\ \mathrm{C} & -1.2668101482 & -0.2054048406 & -0.4509131614 \\ \mathrm{C} & 0.2645783671 & -0.6598029717 & 1.379423417 \\ \mathrm{H} & -3.1062789776 & 1.3362561984 & 1.624505295 \\ \mathrm{C} & 0.0399702944 & 2.7643009172 & 1.352353224 \\ \mathrm{H} & -1.906503266 & 2.5248271526 & -0.9111060341 \\ \mathrm{C} & -3.7716755326 & -0.3791830881 & -0.4408883536 \\ \mathrm{C} & 0.9095235943 & 1.0493586317 & -0.1034083388 \\ \mathrm{H} & -2.5803992164 & 2.9894364887 & 1.4779777844 \\ \mathrm{H} & -0.9334324459 & 1.2080445178 & 2.4958075947 \\ \mathrm{H} & -3.6185017077 & 2.3075085984 & -0.6416182044 \\ \mathrm{C} & -0.88838716 & -1.3551619862 & -0.9876118531 \\ \mathrm{H} & -3.7137925855 & -1.3622588662 & -0.8839841378 \\ \mathrm{H} & -4.6920411171 & 0.0969824855 & -0.7632979118 \\ \mathrm{H} & -3.7947541605 & -0.4826485838 & 0.6360450206 \\ \mathrm{C} & 0.1587113777 & 3.5785237829 & 2.6465114956 \\ \mathrm{H} & -0.2958261617 & 3.414092557 & 0.5496079513 \\ \mathrm{C} & 1.3647159397 & 2.1090172226 & 0.9151298069 \\ \mathrm{H} & 2.0663648457 & 2.818994082 & 0.4940519924 \\ \mathrm{H} & 0.513593601 & 2.9475741378 & 3.4544918171 \\ \mathrm{H} & -0.8009172754 & 3.9935464431 & 2.9365060112 \\ \mathrm{H} & 0.8546367558 & 4.4019968773 & 2.5239611937 \\ \mathrm{O} & -1.631336402 & -1.9918415659 & -1.9579722315 \\ \mathrm{C} & 1.2134548742 & -1.3666082727 & 0.4215386742 \\ \mathrm{C} & 2.1431932963 & -2.3332036952 & 1.1438724634 \\ \mathrm{C} & 1.9009757154 & -0.1370427712 & -0.3057186067 \\ \mathrm{C} & 0.3409549265 & -2.0612476048 & -0.5939516037 \\ & & & \\ & & & \end{array}$




$\begin{array}{llll}\mathrm{O} & 0.6092539619 & -3.1374130499 & -1.0988981034 \\ \mathrm{C} & 2.3270168625 & -0.4433561119 & -1.7379092061 \\ \mathrm{H} & 2.8039403806 & 0.0744403113 & 0.2563592165 \\ \mathrm{H} & 2.7247098631 & -2.9167229569 & 0.4485170601 \\ \mathrm{H} & 1.5604960302 & -3.0102617604 & 1.7553265212 \\ \mathrm{H} & 2.8115395931 & -1.7860920735 & 1.7972275386 \\ \mathrm{O} & 0.1070707423 & -0.9141490165 & 2.5455799106 \\ \mathrm{H} & -1.1821154755 & -2.8107487285 & -2.2193631557 \\ \mathrm{C} & 1.7720178021 & 0.0747008245 & -2.8163995423 \\ \mathrm{C} & 3.46781189 & -1.4392619065 & -1.8652880932 \\ \mathrm{H} & 0.9433943126 & 0.7503970759 & -2.7925376532 \\ \mathrm{H} & 2.1329310298 & -0.1817673539 & -3.7944511905 \\ \mathrm{H} & 3.8947399082 & -1.4075310242 & -2.8594406319 \\ \mathrm{H} & 3.0992230023 & -2.4426401969 & -1.6881122242 \\ \mathrm{H} & 4.2536215997 & -1.2295657086 & -1.1473975608 \\ \mathrm{H} & 0.6526402541 & 1.5343604416 & -1.0293399954 \\ \mathrm{H} & 1.8302994862 & 1.6295246695 & 1.7721946171\end{array}$

$H F=-991.5537839$ 
Carbon

mPW1PW91/

Number

Exptl shift $\quad 6-31 G(d, p)$

corrected difference |difference|

$61.5 \quad 63.3$

133.4

64.2

141.5

28.4

31.6

137.7

$-2.7$

2.7

24.2

31.0

3.8

3.8

25.8

24.9

$-2.6$

2.6

19.2

21.6

20.5

$-0.7$

0.7

40.1

40.5

40.3

1.3

41.5

43.5

43.5

$-1.3$

0.2

42.9

41.3

41.2

2.0

54.9

52.0

52.4

1.7

53.6

54.1

139.9

142.5

114.7

22.7

203.8

70.1

108.5

25.9

200.1

67.9

$-0.2$

2.5

144.5

1.6

144.5

2.0

111.6

25.0

207.6

3.1

3.1

2.3

$-2.3$

3.8

186.7

69.1

$-3.8$

1.0

193.0

193.6

1.0

0.6

140.3

145.0

$-0.6$

1.1

16.5

15.2

1.1

2.4

18.1

17.4

2.4

0.7

16.3

15.0

$-1.7$

1.7

average

1.9

max

3.8

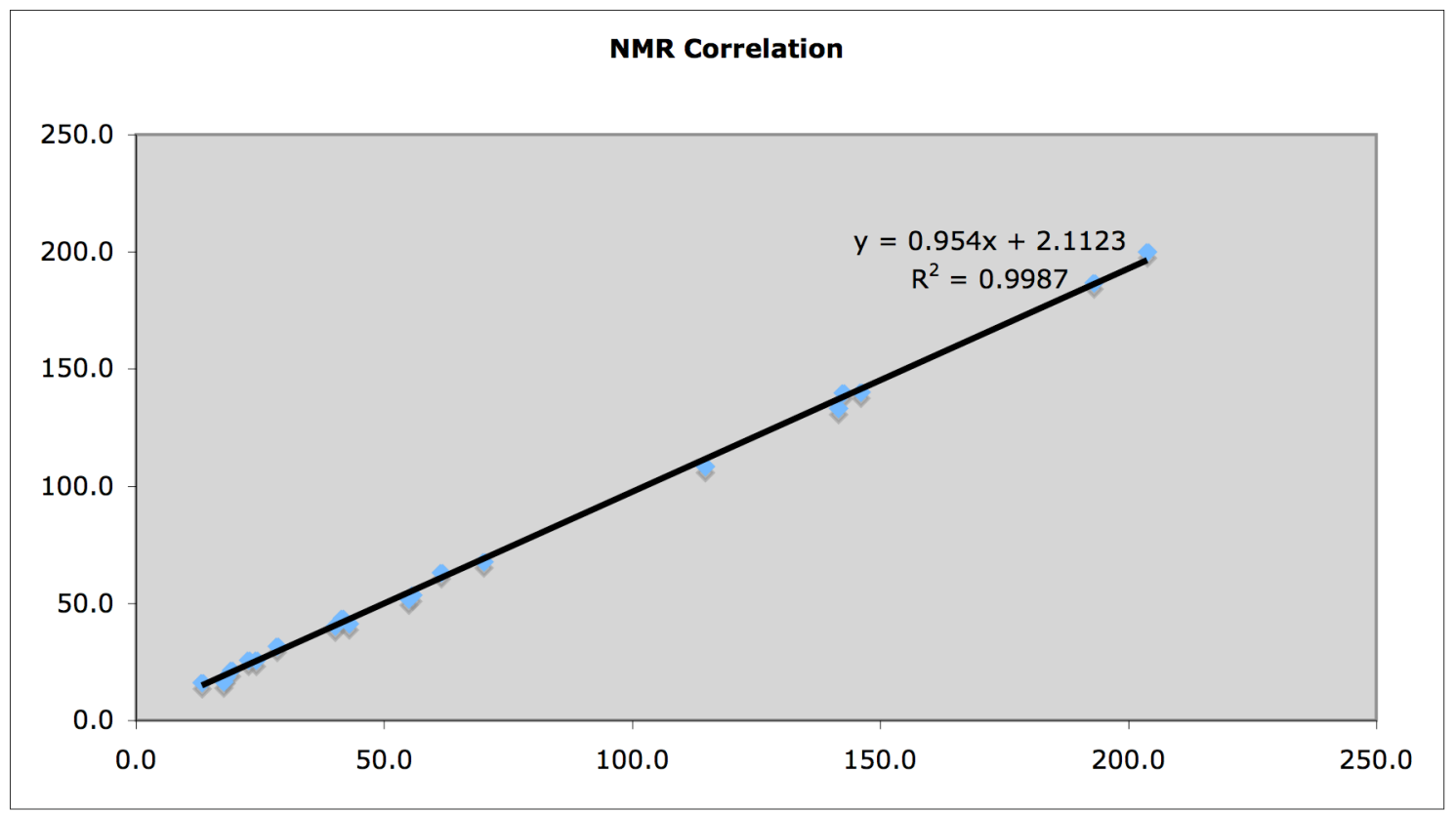


Maoecrystal V structure optimized at HF/3-21G

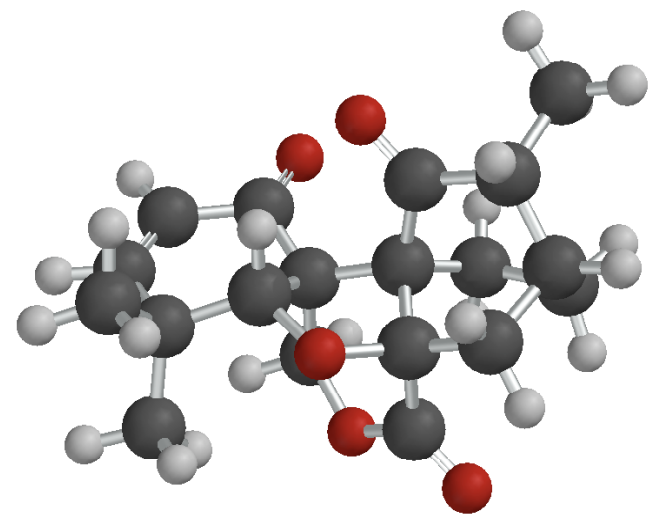

\begin{tabular}{|c|c|c|c|}
\hline $\mathrm{H}$ & -0.5430197571 & -1.3772870373 & -1.2726274818 \\
\hline $\mathrm{C}$ & -0.5426212388 & -1.3766587112 & -0.1958581297 \\
\hline $\mathrm{C}$ & 1.9271255837 & -1.3770668065 & -0.402928014 \\
\hline $\mathrm{C}$ & 0.75455021 & -0.6699196531 & 0.2247630181 \\
\hline $\mathrm{C}$ & -0.6954379757 & -2.828878932 & 0.2391843093 \\
\hline $\mathrm{C}$ & 0.3935416355 & 0.7655597573 & -0.1538310609 \\
\hline $\mathrm{C}$ & -1.7060921805 & -3.5155588057 & -0.7145366307 \\
\hline $\mathrm{O}$ & -1.6002158169 & -0.4810342977 & 0.2519445373 \\
\hline $\mathrm{C}$ & 1.0389282917 & -0.6949381184 & 1.7347336775 \\
\hline $\mathrm{C}$ & -1.2252561568 & -3.0007558268 & 1.6776422855 \\
\hline $\mathrm{H}$ & -1.8481953217 & -4.5529653598 & -0.4330800722 \\
\hline $\mathrm{H}$ & -1.3566174472 & -3.4787522862 & -1.739814123 \\
\hline $\mathrm{H}$ & -2.6599762504 & -3.0051423314 & -0.6519717479 \\
\hline $\mathrm{H}$ & -1.2635355323 & -4.0538353288 & 1.9328722984 \\
\hline $\mathrm{H}$ & -2.2282949424 & -2.5976134634 & 1.7195273946 \\
\hline $\mathrm{H}$ & -0.6411619874 & -2.4824542838 & 2.4182634816 \\
\hline $\mathrm{H}$ & 1.9651439649 & -0.1720443287 & 1.9195392302 \\
\hline $\mathrm{H}$ & 1.1334340197 & -1.6966474157 & 2.1040592645 \\
\hline $\mathrm{O}$ & 0.0027673047 & -0.0593749076 & 2.5225076447 \\
\hline $\mathrm{C}$ & 1.2342718941 & 1.9627130126 & 0.3420180212 \\
\hline $\mathrm{C}$ & -0.8229829444 & 0.8893810476 & 2.0127498538 \\
\hline $\mathrm{C}$ & -1.0052295287 & 0.8254564391 & 0.5016864288 \\
\hline $\mathrm{O}$ & -1.3681894046 & 1.6729109378 & 2.7360479445 \\
\hline $\mathrm{C}$ & -0.8451081332 & 2.9853112479 & -0.7207142872 \\
\hline $\mathrm{C}$ & 0.3597932694 & 3.253310254 & 0.2116237015 \\
\hline $\mathrm{H}$ & -0.0109970814 & 3.5417299823 & 1.1870335879 \\
\hline $\mathrm{H}$ & 0.958587807 & 4.0702849734 & -0.1665012536 \\
\hline $\mathrm{H}$ & 2.1401970913 & 2.0227399299 & -0.2462396286 \\
\hline $\mathrm{H}$ & 1.5292405564 & 1. 8299251849 & 1.3732119169 \\
\hline $\mathrm{C}$ & -1.8208512951 & 2.0037354977 & -0.0257671386 \\
\hline $\mathrm{C}$ & -0.3694055646 & 2.314121824 & -2.0162108483 \\
\hline $\mathrm{H}$ & -1.3542865255 & 3.9130222535 & -0.9476535331 \\
\hline
\end{tabular}




$\begin{array}{llll}\mathrm{C} & 0.2476018636 & 0.9746426286 & -1.6530924436 \\ \mathrm{O} & 0.60412004 & 0.1670175173 & -2.4822189798 \\ \mathrm{C} & 0.6098053994 & 3.144753482 & -2.8696327433 \\ \mathrm{H} & -1.2353053794 & 2.092538026 & -2.6336594639 \\ \mathrm{C} & 0.6598085183 & -3.478138999 & 0.0011300621 \\ \mathrm{H} & 0.6645449815 & -4.5514252684 & 0.0699205333 \\ \mathrm{O} & 2.9371625087 & -0.8193438352 & -0.7698914893 \\ \mathrm{C} & 1.7791258121 & -2.8555503492 & -0.3470951628 \\ \mathrm{H} & 2.666197873 & -3.4110763121 & -0.5775577077 \\ \mathrm{H} & -2.5468096535 & 1.6242225772 & -0.7333176244 \\ \mathrm{H} & -2.3410882309 & 2.4797233223 & 0.7914775203 \\ \mathrm{H} & 0.8833110068 & 2.580770842 & -3.7519877349 \\ \mathrm{H} & 1.5139948232 & 3.3824767219 & -2.3244261927 \\ \mathrm{H} & 0.1400741702 & 4.0712923501 & -3.1793753148\end{array}$

$H F=-1100.4751954$ 
Carbon

Number

mPW1PW91/

\begin{tabular}{rr}
\multicolumn{1}{r}{ Exptl shift } \\
1 & 194.8 \\
2 & 127.2 \\
3 & 156.7 \\
4 & 38.3 \\
5 & 85.5 \\
7 & 169.5 \\
8 & 84.6 \\
9 & 56.9 \\
10 & 52.4 \\
11 & 18.7 \\
12 & 18.2 \\
13 & 32.9 \\
14 & 34.9 \\
15 & 211.7 \\
16 & 48.3 \\
17 & 15.0 \\
18 & 30.4 \\
19 & 18.4 \\
20 & 69.5
\end{tabular}

corrected difference |difference|

189

123.0

148.9

40.7

83.5

164.9

83.5

57.9

53.7

19.4

19.0

33.9

33.7

207.8

48.6

16.7

30.0

19.7

71.1
194.8

126.0

153.0

40.3

84.9

169.6

84.9

58.2

53.9

18.1

17.7

33.2

33.0

214.3

48.5

15.3

29.2

18.5

72.0
0.0

1.2

3.7

$-2.0$

0.6

$-0.1$

$-0.3$

$-1.3$

$-1.5$

0.6

0.5

$-0.3$

1.9

$-2.6$

$-0.2$

$-0.3$

1.2

$-0.1$

$-2.5$

average

max
0.0

1.2

3.7

2.0

0.6

0.1

0.3

1.3

1.5

0.6

0.5

0.3

1.9

2.6

0.2

0.3

1.2

0.1

2.5

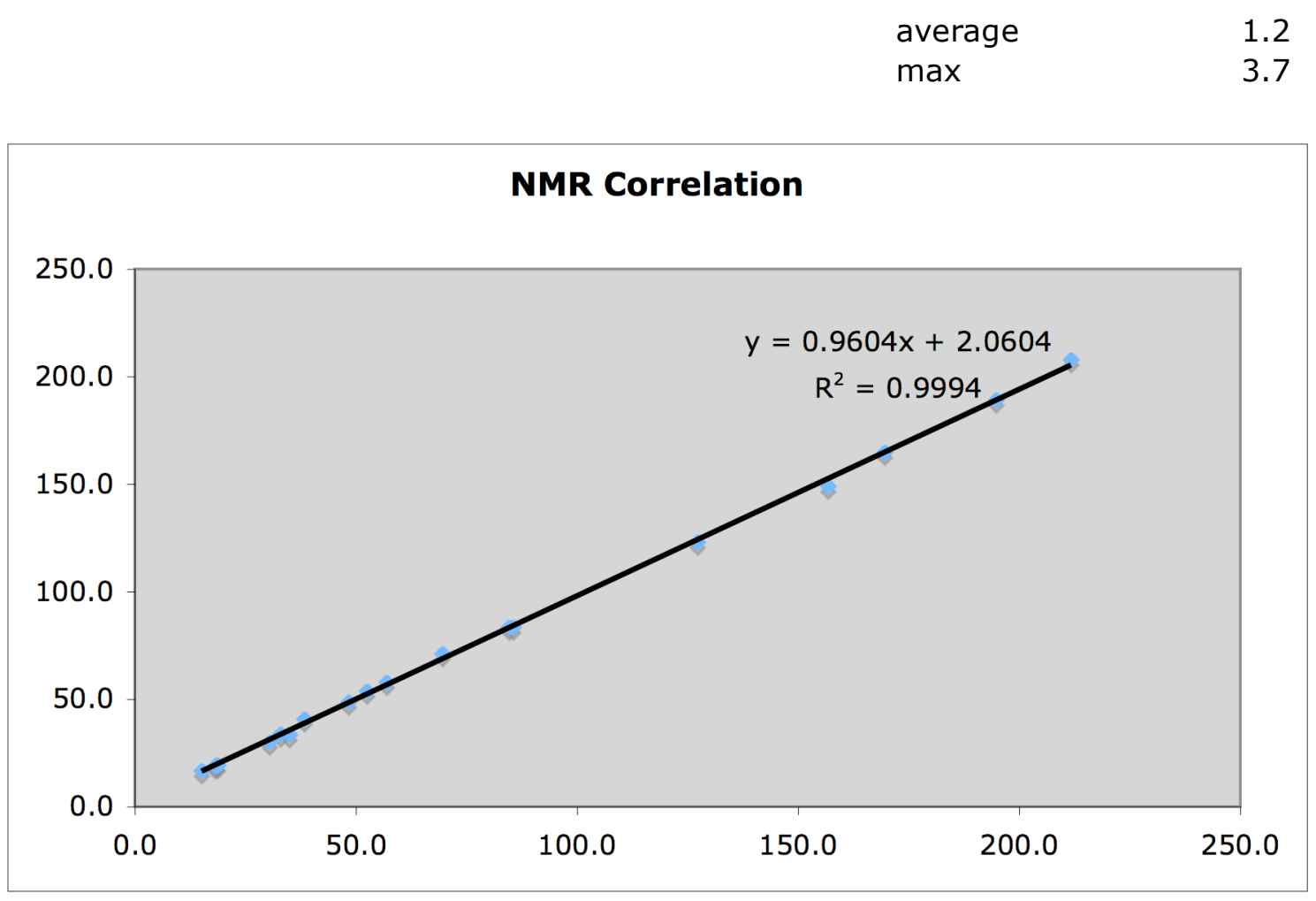


Elisabethin A structure optimized at $\mathrm{HF} / 3-21 \mathrm{G}$

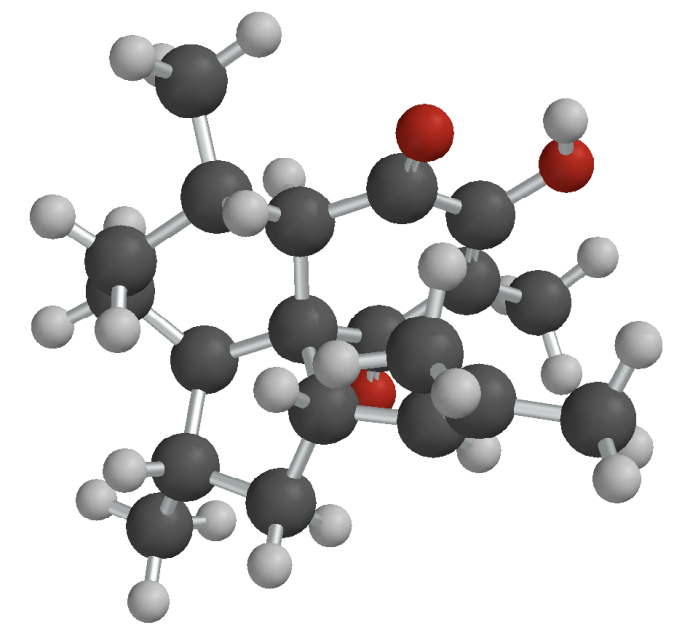

$\begin{array}{llll}\mathrm{H} & -2.465026865 & 0.4433020121 & -0.9806759615 \\ \mathrm{C} & -2.464443301 & 0.4454295816 & 0.1006560714 \\ \mathrm{C} & 0.0670438946 & 0.4457286801 & 0.6971112699 \\ \mathrm{C} & -1.5807506311 & 2.0119454963 & 1.908343332 \\ \mathrm{C} & -0.0991699576 & 1.6762410143 & 1.6464546729 \\ \mathrm{C} & -2.4128860986 & 1.9079176079 & 0.6210750773 \\ \mathrm{C} & -1.243477136 & -0.3606848977 & 0.5933000742 \\ \mathrm{H} & -1.9909748247 & 1.3402272231 & 2.6566343054 \\ \mathrm{H} & -1.9875419571 & 2.5631783576 & -0.1293914069 \\ \mathrm{H} & -1.6455692098 & 3.0147990868 & 2.3183955995 \\ \mathrm{H} & 0.3823685841 & 1.4478050157 & 2.5852527765 \\ \mathrm{H} & -3.4242210642 & 2.2540583127 & 0.8017364463 \\ \mathrm{H} & -1.4621020798 & -0.716487033 & 1.5999466374 \\ \mathrm{C} & -3.7603944489 & -0.2447573429 & 0.5743227597 \\ \mathrm{H} & -3.7957711667 & -1.2685648503 & 0.2276742185 \\ \mathrm{H} & -4.6270694301 & 0.2758310573 & 0.1822229707 \\ \mathrm{H} & -3.8209319626 & -0.2345030761 & 1.6584030064 \\ \mathrm{C} & 0.6704634664 & 2.858256764 & 0.9775227547 \\ \mathrm{H} & -0.0482596866 & 3.5665276937 & 0.579035688 \\ \mathrm{C} & 1.5939435567 & 3.5756499194 & 1.972323513 \\ \mathrm{H} & 2.3035160552 & 2.8643603987 & 2.3786846898 \\ \mathrm{H} & 1.0264541382 & 4.0061347071 & 2.7917567152 \\ \mathrm{H} & 2.1413494371 & 4.377037509 & 1.4863124687 \\ \mathrm{C} & 1.4415867991 & 2.2269132408 & -0.1981440905 \\ \mathrm{H} & 2.3833958511 & 1.8276231359 & 0.1561998933 \\ \mathrm{H} & 1.6381603362 & 2.9320798981 & -0.9963387344 \\ \mathrm{C} & 0.5363162602 & 1.0715600245 & -0.6689232584 \\ \mathrm{H} & -0.3405119447 & 1.4977546054 & -1.1308212206 \\ \mathrm{C} & -1.0144703452 & -1.6059362187 & -0.2090916823 \\ & & & \\ & & & \end{array}$




$\begin{array}{llll}\mathrm{C} & 0.1977226597 & -2.389040832 & 0.1482238998 \\ \mathrm{C} & 1.1820737349 & -1.8793447046 & 0.8673914491 \\ \mathrm{O} & -1.7746297129 & -2.071082206 & -1.0405228676 \\ \mathrm{O} & 0.2081275893 & -3.6606465051 & -0.3352226122 \\ \mathrm{H} & -0.5652415349 & -3.8046096976 & -0.9037493836 \\ \mathrm{C} & 2.3900625955 & -2.6739477607 & 1.2739580674 \\ \mathrm{H} & 2.284358979 & -3.7028551543 & 0.9668530019 \\ \mathrm{H} & 2.5194551574 & -2.6286382125 & 2.348736552 \\ \mathrm{H} & 3.2843364646 & -2.2569072687 & 0.824068647 \\ \mathrm{C} & 1.1799395112 & -0.4472721544 & 1.2348279008 \\ \mathrm{O} & 2.0919561717 & 0.0056444032 & 1.904823484 \\ \mathrm{C} & 1.2255162684 & 0.1356397983 & -1.6242266295 \\ \mathrm{H} & 2.1878191192 & -0.2101875112 & -1.288068156 \\ \mathrm{C} & 0.8018301541 & -0.2709944543 & -2.8096286114 \\ \mathrm{C} & 1.6346541109 & -1.2208956116 & -3.6496125916 \\ \mathrm{H} & 1.8865722224 & -0.7618288988 & -4.6011987687 \\ \mathrm{H} & 1.0724118957 & -2.1262252773 & -3.8616345576 \\ \mathrm{H} & 2.552474952 & -1.4986550629 & -3.1474502133 \\ \mathrm{C} & -0.5281677299 & 0.0888749334 & -3.4427384584 \\ \mathrm{H} & -0.9770551289 & 0.9696444018 & -3.006815343 \\ \mathrm{H} & -1.2216172325 & -0.738428572 & -3.3226095678 \\ \mathrm{H} & -0.3987716698 & 0.2685031599 & -4.5046574575\end{array}$

$H F=-992.717551$ 


\begin{tabular}{|c|c|c|c|c|c|}
\hline \multicolumn{6}{|c|}{ mPW1PW91/ } \\
\hline Number & Exptl shift & $6-31 G(d, p)$ & corrected & difference & |difference| \\
\hline 1 & 66.3 & 68.6 & 69.0 & -2.7 & 2.7 \\
\hline 2 & 53.4 & 53.3 & 53.2 & 0.2 & 0.2 \\
\hline 3 & 24.4 & 27.1 & 26.1 & -1.7 & 1.7 \\
\hline 4 & 25.3 & 25.0 & 24.0 & 1.3 & 1.3 \\
\hline 5 & 24.0 & 25.0 & 24.0 & 0.0 & 0.0 \\
\hline 6 & 45.8 & 47.6 & 47.3 & -1.5 & 1.5 \\
\hline 7 & 38.0 & 40.1 & 39.6 & -1.6 & 1.6 \\
\hline 8 & 38.8 & 38.5 & 37.9 & 0.9 & 0.9 \\
\hline 9 & 47.2 & 49.6 & 49.4 & -2.2 & 2.2 \\
\hline 10 & 127.3 & 124.4 & 126.6 & 0.7 & 0.7 \\
\hline 11 & 132.8 & 129.9 & 132.3 & 0.5 & 0.5 \\
\hline 12 & 17.7 & 17.9 & 16.6 & 1.1 & 1.1 \\
\hline 13 & 25.9 & 25.6 & 24.6 & 1.3 & 1.3 \\
\hline 14 & 202.5 & 197.1 & 201.7 & 0.8 & 0.8 \\
\hline 15 & 120.1 & 116.4 & 118.4 & 1.7 & 1.7 \\
\hline 16 & 155.8 & 149.9 & 153.0 & 2.8 & 2.8 \\
\hline 17 & 195.9 & 195.1 & 199.7 & -3.8 & 3.8 \\
\hline 18 & 22.6 & 21.9 & 20.8 & 1.8 & 1.8 \\
\hline 19 & 18.9 & 19.1 & 17.9 & 1.0 & 1.0 \\
\hline 20 & 8.3 & 11.4 & 9.9 & -1.6 & 1.6 \\
\hline & & & & $\begin{array}{l}\text { average } \\
\max \end{array}$ & $\begin{array}{l}1.4 \\
3.8\end{array}$ \\
\hline
\end{tabular}

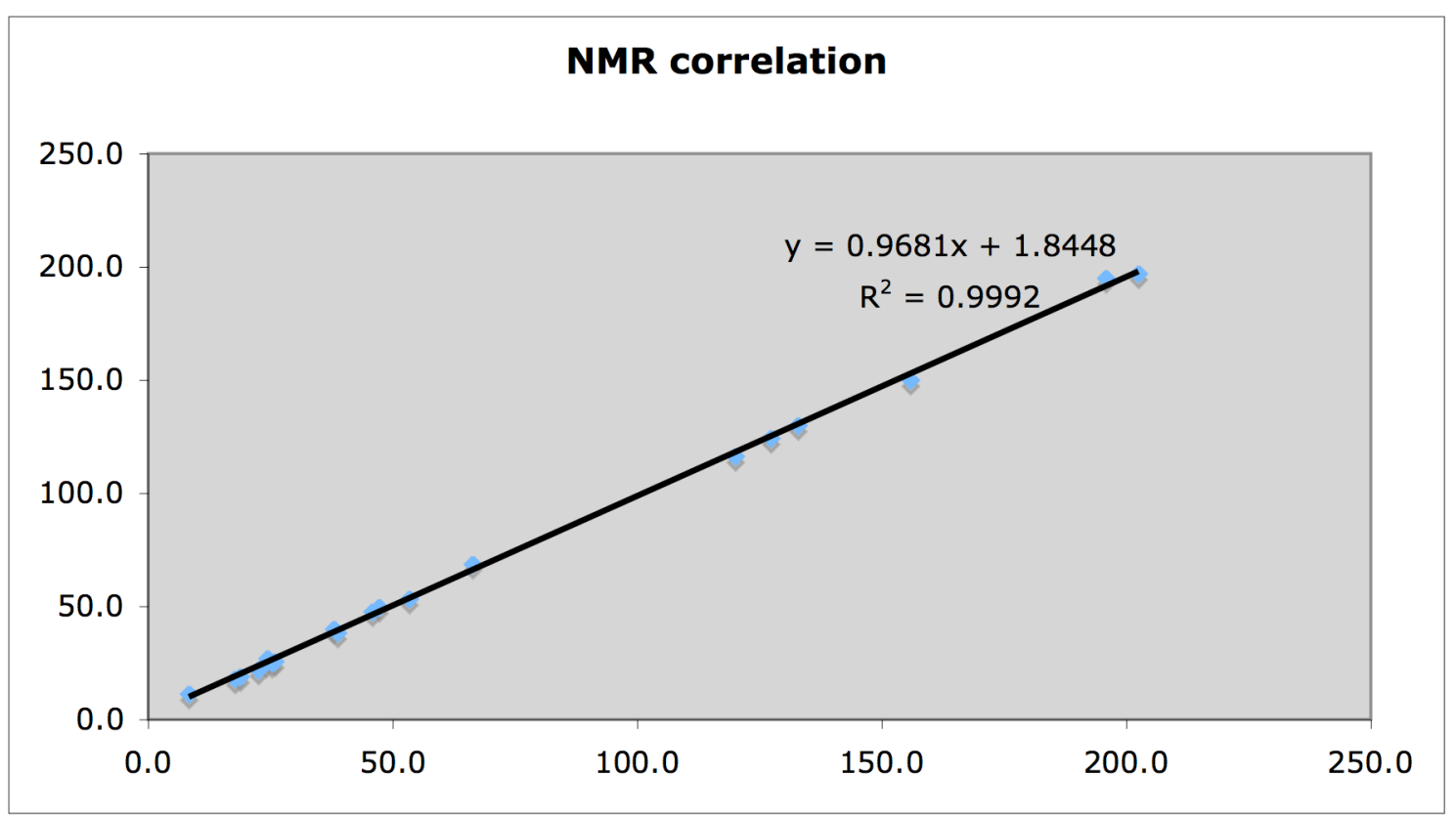


Hexacyclinol 7 conformation 1 optimized at HF/3-21G

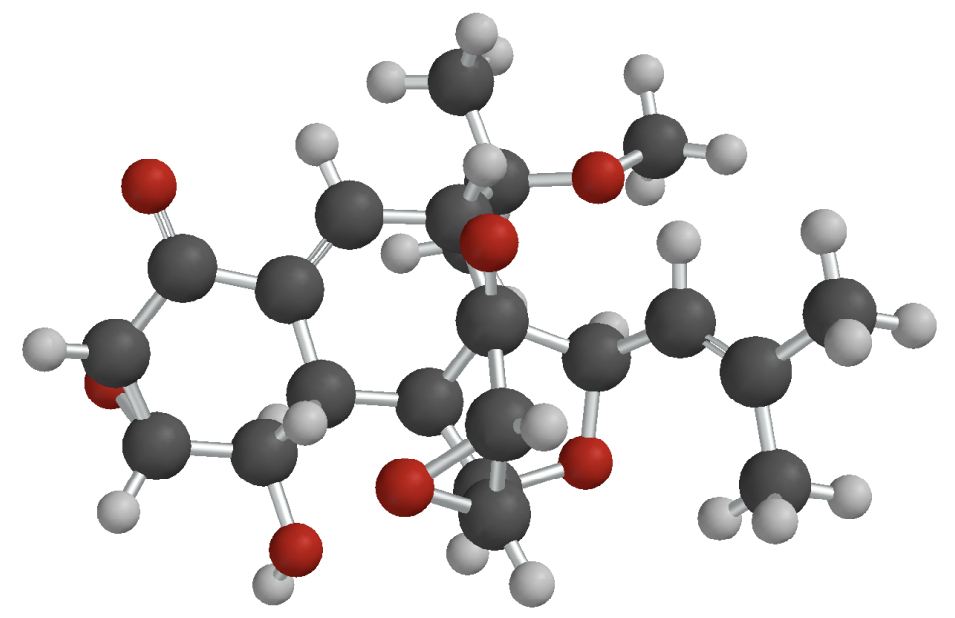

$\begin{array}{llll}\mathrm{C} & 1.7513062766 & 0.4851768621 & 0.7445718902 \\ \mathrm{C} & 1.7495687302 & 0.4867779872 & 3.3151688811 \\ \mathrm{C} & 3.926169701 & 0.4744796861 & 1.9628525067 \\ \mathrm{C} & 3.214749307 & 0.7763447851 & 3.212118904 \\ \mathrm{C} & 3.1513378713 & -0.1215405 & 0.8080099938 \\ \mathrm{C} & 1.0366021354 & 0.178199456 & 2.0451241289 \\ \mathrm{H} & 4.8118330862 & 1.0240953958 & 1.7191531952 \\ \mathrm{H} & 3.0796089971 & -1.190143546 & 0.9702606301 \\ \mathrm{H} & 3.590143519 & 1.5038554807 & 3.8997028681 \\ \mathrm{C} & 0.9604845999 & -0.1004568966 & -0.4189637653 \\ \mathrm{H} & 1.1610638892 & -1.1636945614 & -0.4522833004 \\ \mathrm{C} & -0.5678512051 & 0.0572590249 & -0.3005165206 \\ \mathrm{C} & -0.2060435477 & -0.2647406871 & 2.1351926102 \\ \mathrm{H} & -0.6021669247 & -0.3849103796 & 3.1244621157 \\ \mathrm{C} & -1.1432277926 & -0.5693759758 & 0.9884107146 \\ \mathrm{O} & 4.0439240403 & -0.445096902 & 3.105211101 \\ \mathrm{C} & -0.9347091142 & 1.5452466923 & -0.2938522455 \\ \mathrm{C} & 1.1904313164 & 0.4069091691 & -1.8408711874 \\ \mathrm{H} & 2.1431479636 & 0.1160073748 & -2.2358172315 \\ \mathrm{C} & 0.9004850888 & 1.8706887426 & -2.03259282 \\ \mathrm{H} & 1.1362535745 & 2.2842258477 & -2.9903228422 \\ \mathrm{C} & -0.2305546884 & 2.4198497012 & -1.2859306966 \\ \mathrm{H} & -0.8059339948 & 3.2397612849 & -1.6569657272 \\ \mathrm{O} & 3.7927026475 & 0.1770994391 & -0.4476436197 \\ \mathrm{H} & 4.6075756197 & -0.3318856967 & -0.5542663865 \\ \mathrm{O} & 0.134137819 & -0.268969838 & -2.5796102596 \\ \mathrm{O} & 1.1596429218 & 2.7736754681 & -0.905802433 \\ \mathrm{O} & 1.1970278181 & 0.51009237 & 4.3954496799 \\ \mathrm{O} & -1.7958783209 & 2.014976422 & 0.4142392529\end{array}$




$\begin{array}{llll}\mathrm{C} & -1.01784111 & -0.4996761877 & -1.7062511043 \\ \mathrm{H} & 1.8509411418 & 1.5514326773 & 0.6162738125 \\ \mathrm{H} & -2.0631868869 & -0.0395057876 & 1.1956136162 \\ \mathrm{C} & -1.5667067512 & -2.067981719 & 0.9864895682 \\ \mathrm{C} & -2.2025140574 & -2.4229739764 & 2.3432584543 \\ \mathrm{H} & -2.6226329786 & -3.4200683126 & 2.3079017385 \\ \mathrm{H} & -2.9969241615 & -1.7251807857 & 2.5782895474 \\ \mathrm{H} & -1.4673964035 & -2.4100974637 & 3.1353035735 \\ \mathrm{C} & -0.4283865737 & -3.0525752784 & 0.6864672548 \\ \mathrm{H} & -0.7524495778 & -4.0740845268 & 0.8467606587 \\ \mathrm{H} & 0.401034964 & -2.8599339172 & 1.3540887794 \\ \mathrm{H} & -0.0857588231 & -2.9666518782 & -0.3347276793 \\ \mathrm{O} & -2.5945192383 & -2.091904989 & -0.0374108232 \\ \mathrm{C} & -3.2735200707 & -3.3206201868 & -0.3493053982 \\ \mathrm{H} & -3.9258397132 & -3.090604548 & -1.1776715958 \\ \mathrm{H} & -3.8702373374 & -3.6745866185 & 0.4804885501 \\ \mathrm{H} & -2.5846439903 & -4.0993339368 & -0.6501079964 \\ \mathrm{H} & -1.1465689627 & -1.5595276799 & -1.6257921213 \\ \mathrm{C} & -2.3125210337 & 0.1055669918 & -2.1803149038 \\ \mathrm{H} & -3.0765070272 & -0.025658144 & -1.4367041437 \\ \mathrm{C} & -2.6334894862 & 0.6868912223 & -3.322119978 \\ \mathrm{C} & -1.7311312389 & 0.90239679 & -4.5203215233 \\ \mathrm{H} & -1.6269278129 & 1.9671203466 & -4.7177918983 \\ \mathrm{H} & -2.1911909725 & 0.4588651846 & -5.3993286974 \\ \mathrm{H} & -0.7615975859 & 0.4681103887 & -4.3646868478 \\ \mathrm{C} & -4.0478123145 & 1.1985824942 & -3.5384540097 \\ \mathrm{H} & -4.0325229279 & 2.2609171644 & -3.7655424316 \\ \mathrm{H} & -4.6704460174 & 1.0441200395 & -2.6665311474 \\ \mathrm{H} & -4.503928411 & 0.6918116536 & -4.3842467495 \\ & & & \end{array}$

$H F=-1403.2951487$ 
Original Carbon assignments: C9 and C12 do not fit well.

Carbon Number mPW1PW91/

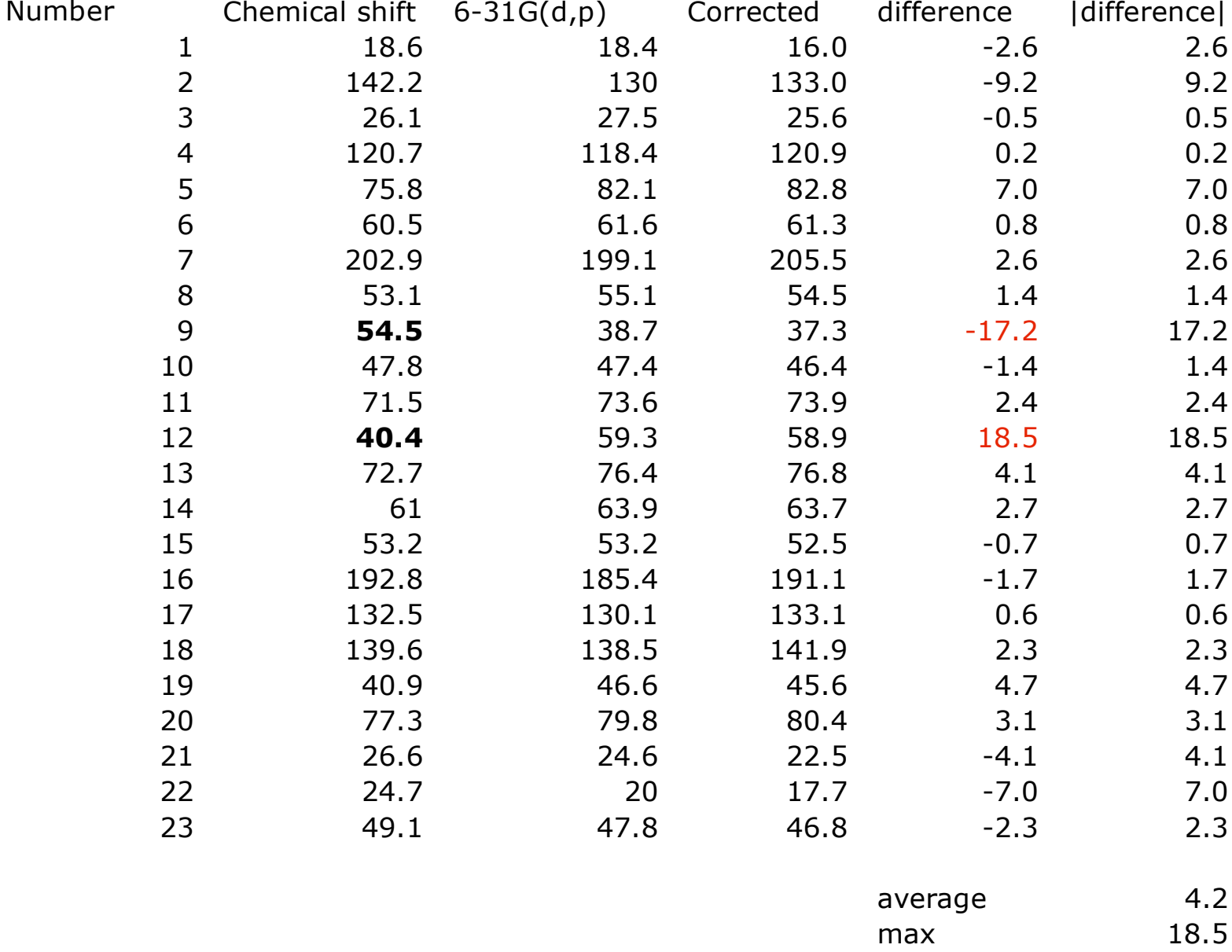

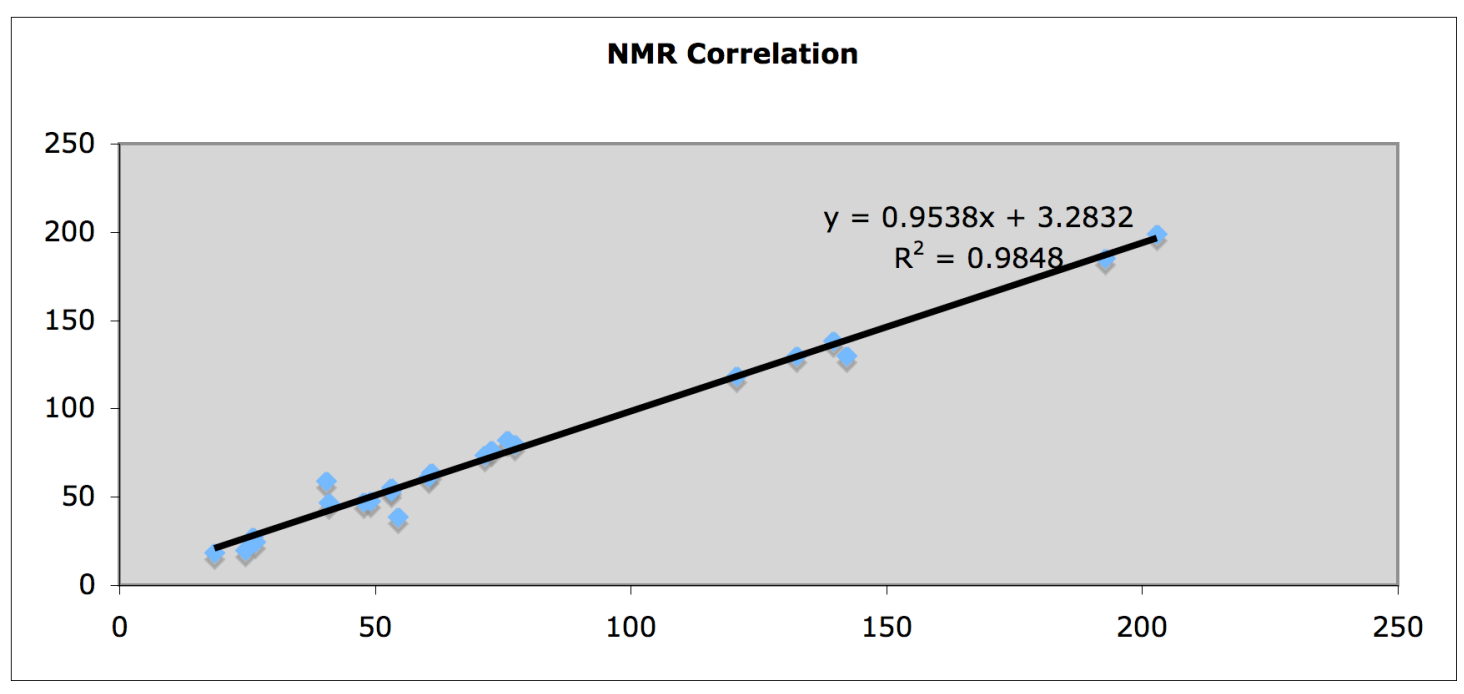


Difference plot for the original ${ }^{13} \mathrm{C}$ assignments with structure 7 , conformation 1 .

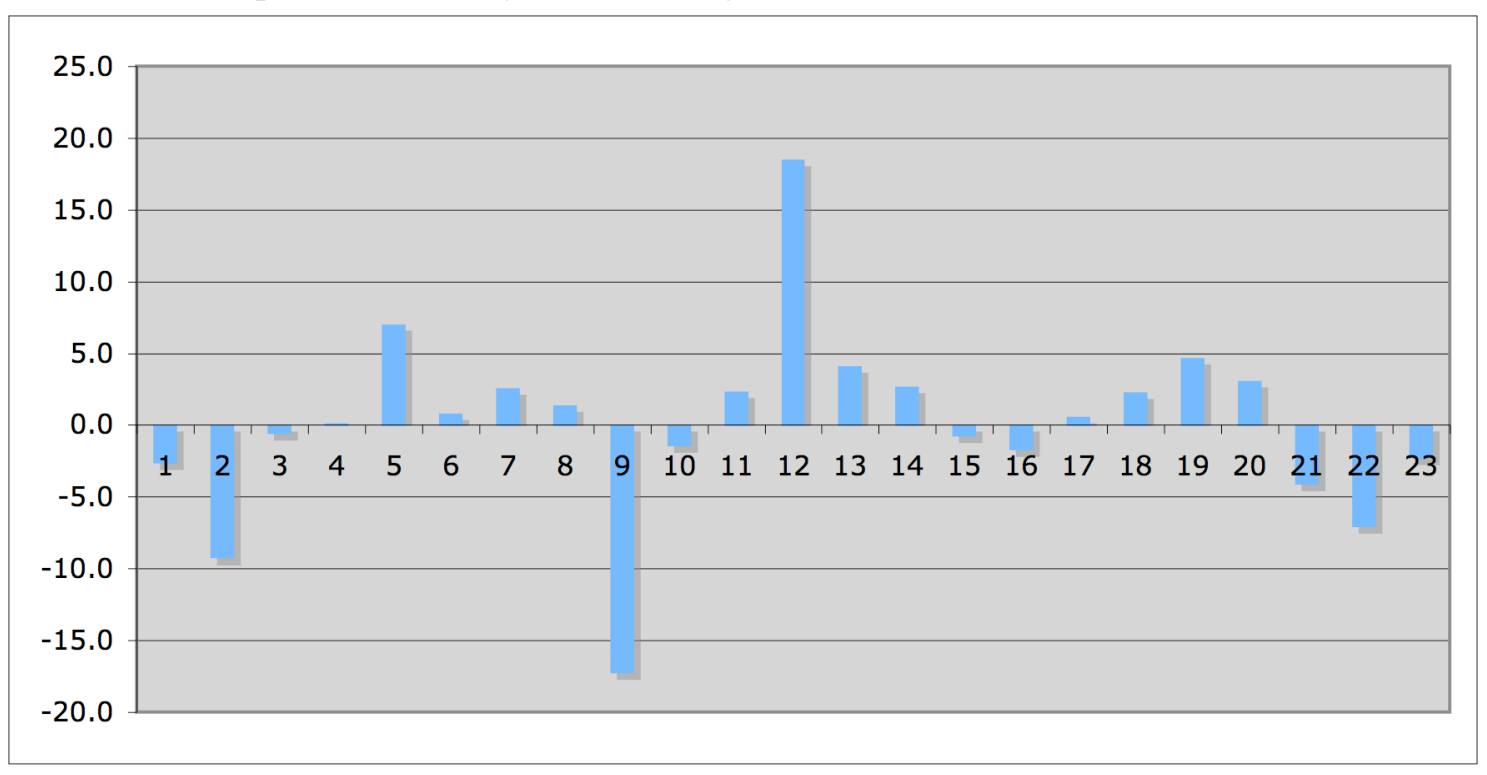




\section{Revised carbon assignments: Exchange $\mathrm{C} 9$ and $\mathrm{C} 12$} mPW1PW91/

Carbon Number
Chemical shift $\quad 6-31 G(d, p)$ 18.6

142.2

26.1

120.7

75.8

60.5

202.9

53.1

40.4

47.8

71.5

$\mathbf{5 4 . 5}$

72.7

61

53.2

192.8

132.5

139.6

40.9

77.3

26.6

24.7

49.1

18.4

130

27.5

118.4

82.1

61.6

199.1

55.1

38.7

47.4

73.6

59.3

76.4

63.9

53.2

185.4

130.1

138.5

46.6

79.8

24.6

20

47.8
Corrected

16.3

132.7

25.8

120.6

82.7

61.4

204.8

54.6

37.5

46.5

73.9

59.0

76.8

63.8

52.6

190.5

132.8

141.6

45.7

80.3

22.8

18.0

47.0 difference

$-2.3$

$-9.5$

$-0.3$

$-0.1$

6.9

0.9

1.9

1.5

$-2.9$

$-1.3$

2.4

4.5

4.1

2.8

$-0.6$

$-2.3$

0.3

2.0

4.8

3.0

$-3.8$

$-6.7$

$-2.1$ |difference|

2.3

9.5

0.3

0.1

6.9

0.9

1.9

1.5

2.9

1.3

2.4

4.5

4.1

2.8

0.6

2.3

0.3

2.0

4.8

3.0

3.8

6.7

2.1

2.9

9.5

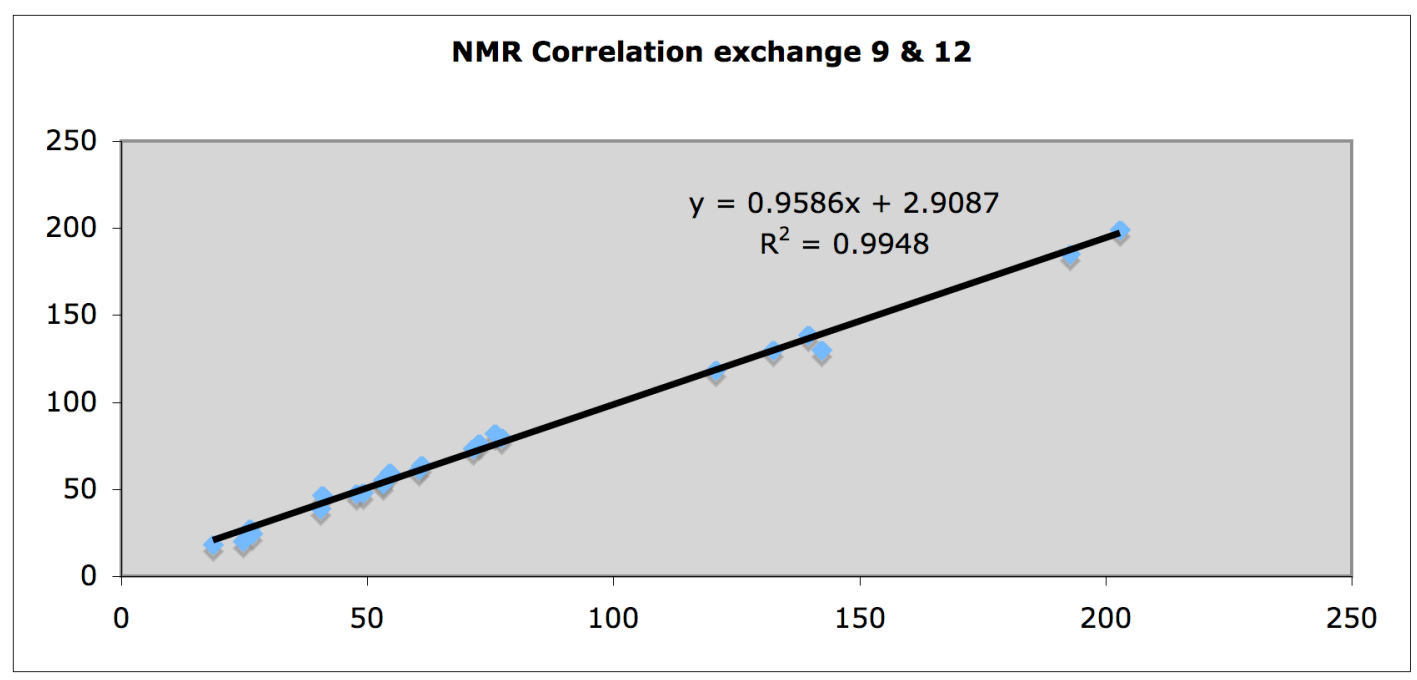


Hexacyclinol 7 conformation 2 optimized at $\mathrm{HF} / 3-21 \mathrm{G}$

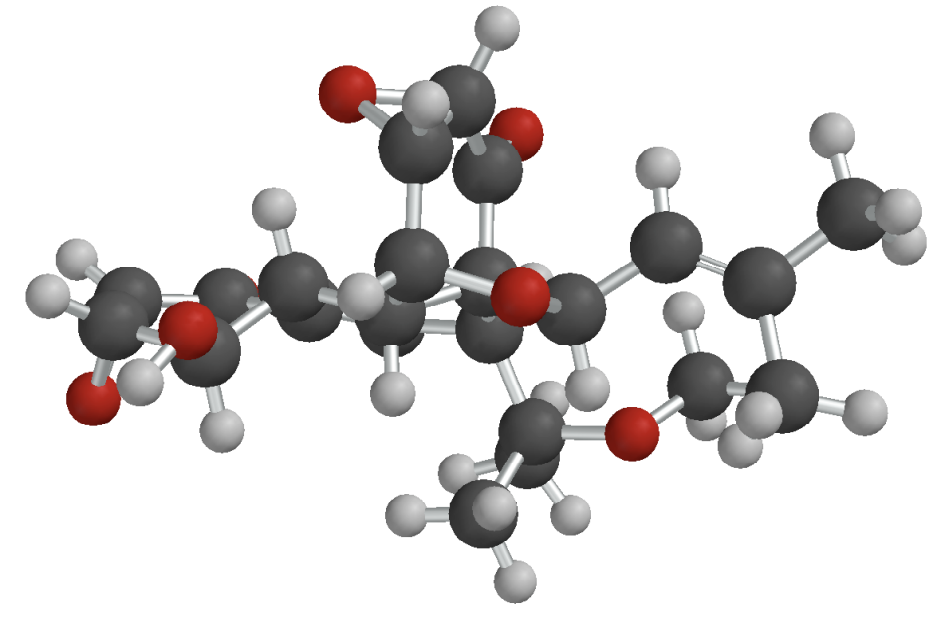

$\begin{array}{llll}\mathrm{C} & 1.8168262765 & 0.692889826 & 0.6821506021 \\ \mathrm{C} & 1.7996353918 & 0.6852765781 & 3.2508625835 \\ \mathrm{C} & 3.9856962115 & 0.712451662 & 1.9128281912 \\ \mathrm{C} & 3.2610641878 & 0.9956172709 & 3.1594030926 \\ \mathrm{C} & 3.2298737242 & 0.1168808167 & 0.7448579109 \\ \mathrm{C} & 1.105351647 & 0.3561050119 & 1.976370952 \\ \mathrm{H} & 4.8670658867 & 1.2743834324 & 1.6819609406 \\ \mathrm{H} & 3.1813613904 & -0.9553744683 & 0.8900559042 \\ \mathrm{H} & 3.6228649554 & 1.7214737791 & 3.8559190377 \\ \mathrm{C} & 1.045609501 & 0.1041709832 & -0.4936980835 \\ \mathrm{H} & 1.2844246453 & -0.9492231072 & -0.5481015484 \\ \mathrm{C} & -0.490170532 & 0.2008028709 & -0.3755176593 \\ \mathrm{C} & -0.1200224334 & -0.1329611918 & 2.0566120381 \\ \mathrm{H} & -0.5195068394 & -0.2731782523 & 3.0419510259 \\ \mathrm{C} & -1.0337564649 & -0.4768162652 & 0.901472227 \\ \mathrm{O} & 4.1048901784 & -0.215392533 & 3.0479827389 \\ \mathrm{C} & -0.9060977185 & 1.6730293671 & -0.3333367478 \\ \mathrm{C} & 1.2577215839 & 0.6447067716 & -1.9055949298 \\ \mathrm{H} & 2.225394317 & 0.4017010931 & -2.2978734066 \\ \mathrm{C} & 0.9167144047 & 2.1014621122 & -2.0666800048 \\ \mathrm{H} & 1.1252348579 & 2.5412673443 & -3.0189458866 \\ \mathrm{C} & -0.2209028459 & 2.6010176728 & -1.2918090383 \\ \mathrm{H} & -0.8198674384 & 3.4184540696 & -1.6327068715 \\ \mathrm{O} & 3.8719163234 & 0.4501979071 & -0.5016497528 \\ \mathrm{H} & 4.6911257311 & -0.0495014817 & -0.6182577782 \\ \mathrm{O} & 0.2317380826 & -0.0474876631 & -2.6622360275 \\ \mathrm{O} & 1.1614381815 & 2.9867426623 & -0.9226355383 \\ \mathrm{O} & 1.2366270829 & 0.7052609221 & 4.3258537436 \\ \mathrm{O} & -1.8095147297 & 2.0890875414 & 0.3573588931 \\ \mathrm{C} & -0.9077521265 & -0.3728855507 & -1.7801045417 \\ \mathrm{H} & 1.8948141181 & 1.7623374682 & 0.5682493397\end{array}$




$\begin{array}{llll}\mathrm{H} & -1.9738999679 & 0.0115340136 & 1.1218745826 \\ \mathrm{C} & -1.3463565562 & -2.0062106009 & 0.8943191962 \\ \mathrm{C} & -1.8699926816 & -2.4403876449 & 2.2793045439 \\ \mathrm{H} & -2.3152444076 & -3.4227039699 & 2.1827307192 \\ \mathrm{H} & -2.6147007994 & -1.7576074244 & 2.6709191142 \\ \mathrm{H} & -1.0583320539 & -2.506138712 & 2.9904037725 \\ \mathrm{C} & -0.1656217297 & -2.9093102871 & 0.5254648143 \\ \mathrm{H} & -0.4745679139 & -3.9415506834 & 0.6313133248 \\ \mathrm{H} & 0.6751245655 & -2.7236630323 & 1.1803216749 \\ \mathrm{H} & 0.1370414069 & -2.7642171547 & -0.4997840855 \\ \mathrm{O} & -2.3460852381 & -2.28828833 & -0.1210225262 \\ \mathrm{C} & -3.7124171645 & -1.8931045327 & 0.1173448571 \\ \mathrm{H} & -4.2483179927 & -2.1213999349 & -0.7892294844 \\ \mathrm{H} & -3.8019100909 & -0.8337591949 & 0.3147529414 \\ \mathrm{H} & -4.1478076207 & -2.4493765007 & 0.9355548976 \\ \mathrm{H} & -0.9615107014 & -1.4356609713 & -1.7054751442 \\ \mathrm{C} & -2.1612211098 & 0.174655514 & -2.3975139258 \\ \mathrm{H} & -2.3163494991 & 1.233319404 & -2.3079936623 \\ \mathrm{C} & -3.0231655819 & -0.5236604081 & -3.1154760893 \\ \mathrm{C} & -2.9063016065 & -2.0158175095 & -3.3825044342 \\ \mathrm{H} & -2.2454512349 & -2.1886665014 & -4.2267186322 \\ \mathrm{H} & -3.8768648559 & -2.4284823399 & -3.6345902769 \\ \mathrm{H} & -2.5247793311 & -2.5461319682 & -2.5227144306 \\ \mathrm{C} & -4.2039924753 & 0.1484378321 & -3.7863738747 \\ \mathrm{H} & -4.160401868 & -0.0097909859 & -4.8602520379 \\ \mathrm{H} & -4.2211445292 & 1.2139881708 & -3.5961540939 \\ \mathrm{H} & -5.1362051883 & -0.2810979384 & -3.4307122592\end{array}$

$H F=-1403.2895075$ 


\section{Revised carbon assignments: Exchange $\mathrm{C} 9$ and $\mathrm{C12}$}

\begin{tabular}{|c|c|c|c|c|c|}
\hline \multicolumn{6}{|c|}{ mPW1PW91/ } \\
\hline Number & Chemical shift & $6-31 G(d, p)$ & Corrected & difference & |difference| \\
\hline 1 & 18.6 & 17.3 & 15.4 & -3.2 & 3.2 \\
\hline 2 & 142.2 & 136.8 & 138.7 & -3.5 & 3.5 \\
\hline 3 & 26.1 & 27.3 & 25.7 & -0.4 & 0.4 \\
\hline 4 & 120.7 & 119.1 & 120.4 & -0.3 & 0.3 \\
\hline 5 & 75.8 & 77.6 & 77.6 & 1.8 & 1.8 \\
\hline 6 & 60.5 & 61 & 60.5 & 0.0 & 0.0 \\
\hline 7 & 202.9 & 202 & 206.0 & 3.1 & 3.1 \\
\hline 8 & 53.1 & 55.1 & 54.4 & 1.3 & 1.3 \\
\hline 9 & 40.4 & 38.8 & 37.6 & -2.8 & 2.8 \\
\hline 10 & 47.8 & 48.5 & 47.6 & -0.2 & 0.2 \\
\hline 11 & 71.5 & 71.9 & 71.8 & 0.3 & 0.3 \\
\hline 12 & 54.5 & 60.2 & 59.7 & 5.2 & 5.2 \\
\hline 13 & 72.7 & 76.4 & 76.4 & 3.7 & 3.7 \\
\hline 14 & 61 & 64 & 63.6 & 2.6 & 2.6 \\
\hline 15 & 53.2 & 53.5 & 52.8 & -0.4 & 0.4 \\
\hline 16 & 192.8 & 185.3 & 188.7 & -4.1 & 4.1 \\
\hline 17 & 132.5 & 129.7 & 131.4 & -1.1 & 1.1 \\
\hline 18 & 139.6 & 139.2 & 141.2 & 1.6 & 1.6 \\
\hline 19 & 40.9 & 40.6 & 39.5 & -1.4 & 1.4 \\
\hline 20 & 77.3 & 79.6 & 79.7 & 2.4 & 2.4 \\
\hline 21 & 26.6 & 27.2 & 25.6 & -1.0 & 1.0 \\
\hline 22 & 24.7 & 25.5 & 23.9 & -0.8 & 0.8 \\
\hline 23 & 49.1 & 48.9 & 48.0 & -1.1 & 1.1 \\
\hline & & & & average & 1.8 \\
\hline & & & & $\max$ & 5.2 \\
\hline
\end{tabular}

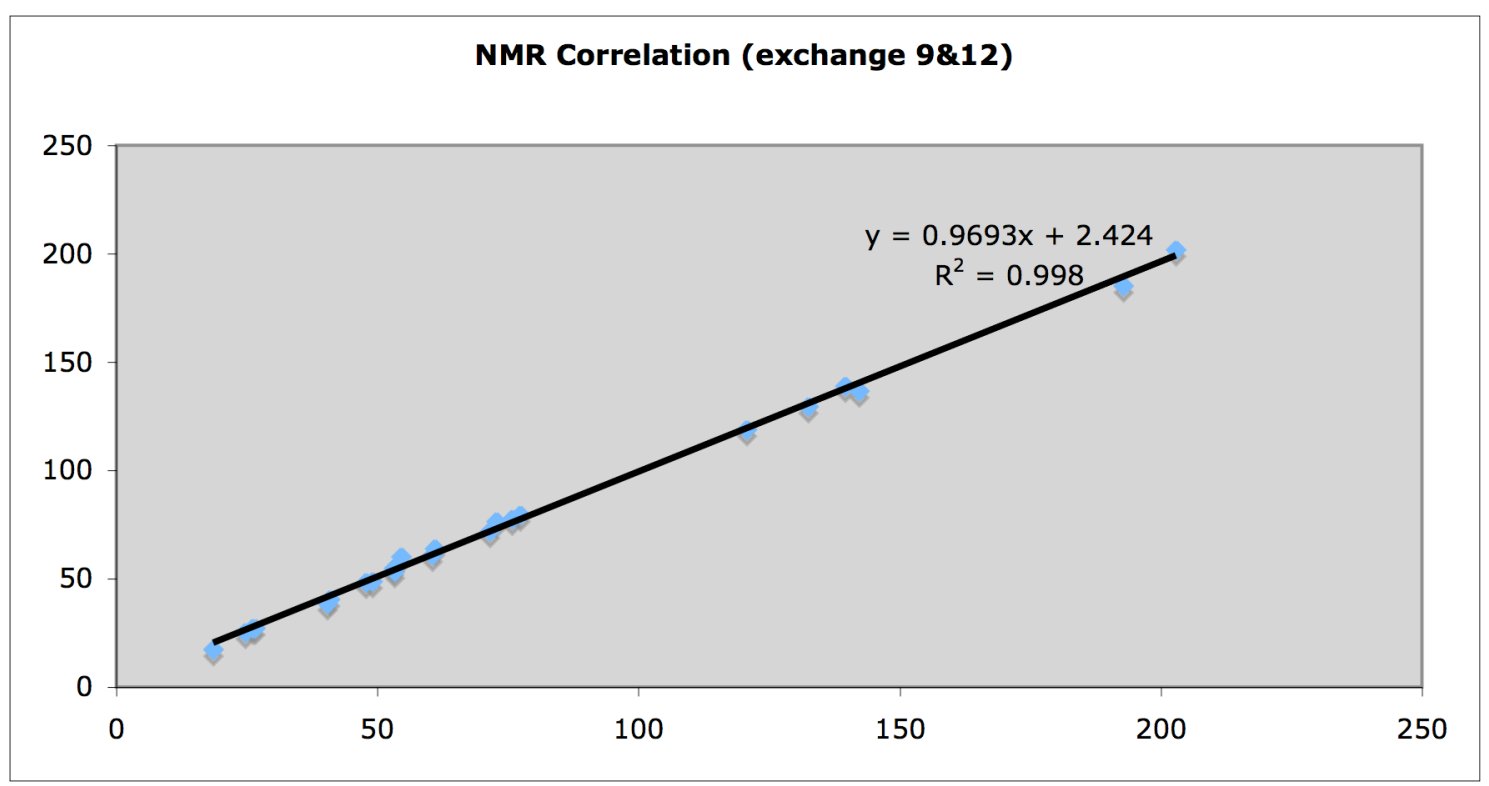


Compound 6-D (5-up \& 19-up) optimized at HF/3-21G

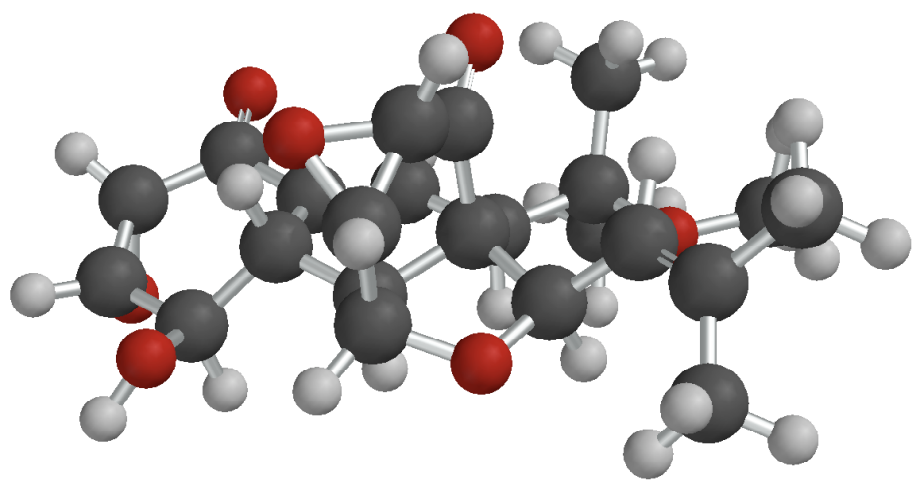

$\begin{array}{llll}\mathrm{C} & 1.8900370926 & 0.4954844881 & 0.6400709891 \\ \mathrm{C} & 1.9031702627 & 0.6696160951 & 3.2033405615 \\ \mathrm{C} & 4.0469768751 & 0.8859957509 & 1.8136784459 \\ \mathrm{C} & 3.3066401579 & 1.1705100084 & 3.0517342741 \\ \mathrm{C} & 3.3630617487 & 0.1005832294 & 0.7143078326 \\ \mathrm{C} & 1.2492907682 & 0.1570716203 & 1.9727012704 \\ \mathrm{H} & 4.8383236734 & 1.5427658073 & 1.5163590547 \\ \mathrm{H} & 3.4486046264 & -0.9527662033 & 0.9513771277 \\ \mathrm{H} & 3.5745294605 & 1.9947225879 & 3.6777366901 \\ \mathrm{C} & 1.1701703253 & -0.2618764803 & -0.471121039 \\ \mathrm{H} & 1.4894553445 & -1.298391489 & -0.4356450057 \\ \mathrm{C} & -0.3763383166 & -0.2750907118 & -0.3188983909 \\ \mathrm{C} & 0.1209401839 & -0.5186991473 & 2.1017910699 \\ \mathrm{H} & -0.2399193862 & -0.6751605418 & 3.0990628706 \\ \mathrm{C} & -0.6672034741 & -1.1099381338 & 0.9502656777 \\ \mathrm{O} & 4.3088288675 & 0.0814722262 & 3.0166712957 \\ \mathrm{C} & -0.8376589953 & 1.1683514079 & -0.2912492699 \\ \mathrm{C} & 1.3150387632 & 0.1584413869 & -1.9276356674 \\ \mathrm{H} & 2.2966847648 & -0.0198975304 & -2.3188429114 \\ \mathrm{C} & 0.8077613383 & 1.5446688988 & -2.2151815235 \\ \mathrm{H} & 0.9162876771 & 1.8941634312 & -3.2200585398 \\ \mathrm{C} & -0.3244351725 & 2.0190485953 & -1.4216350361 \\ \mathrm{H} & -1.022582549 & 2.724885109 & -1.8156714515 \\ \mathrm{O} & 3.9450182887 & 0.4002158664 & -0.5692489486 \\ \mathrm{H} & 4.8235947084 & 0.0063831083 & -0.6556732261 \\ \mathrm{O} & 0.3686442106 & -0.7359342781 & -2.5717053905 \\ \mathrm{O} & 1.0330170716 & 2.5729230499 & -1.1897928649 \\ \mathrm{O} & 1.3581919609 & 0.6846417493 & 4.2876825579 \\ \mathrm{O} & -1.5776757717 & 1.6547365552 & 0.5318251675 \\ \mathrm{C} & -0.7990959617 & -0.9093447844 & -1.7026268017 \\ \mathrm{H} & 1.8383761396 & 1.5561727982 & 0.4502893578 \\ \mathrm{H} & -0.9407936029 & -1.966229088 & -1.5592137299 \\ & & & \\ & & & \end{array}$




$\begin{array}{llll}\mathrm{C} & -2.0594588089 & -0.2998083508 & -2.2706636132 \\ \mathrm{H} & -2.8226672401 & -0.2223422033 & -1.5233625438 \\ \mathrm{C} & -2.3534539106 & 0.0590542469 & -3.5080509244 \\ \mathrm{C} & -1.4661916567 & -0.0542599825 & -4.7311113604 \\ \mathrm{H} & -1.967697627 & -0.6606891666 & -5.4808088541 \\ \mathrm{H} & -0.5147559402 & -0.4898279491 & -4.4908003489 \\ \mathrm{H} & -1.3130053615 & 0.930104215 & -5.1683021592 \\ \mathrm{C} & -3.7276528174 & 0.6287229294 & -3.8209519427 \\ \mathrm{H} & -3.6343892357 & 1.6183941953 & -4.259588531 \\ \mathrm{H} & -4.3456337347 & 0.7032074104 & -2.9350001053 \\ \mathrm{H} & -4.2371515493 & -0.0000682757 & -4.5456278291 \\ \mathrm{H} & -0.2094839311 & -2.070377368 & 0.7262884753 \\ \mathrm{C} & -2.1200988984 & -1.5090132588 & 1.3560477221 \\ \mathrm{C} & -2.9642523451 & -0.420433365 & 2.0302203556 \\ \mathrm{H} & -3.1994413741 & 0.3675742633 & 1.3395072341 \\ \mathrm{H} & -3.879589937 & -0.8509196072 & 2.419028563 \\ \mathrm{H} & -2.4264149186 & 0.0177317666 & 2.8595151866 \\ \mathrm{C} & -2.0220011017 & -2.7342926634 & 2.2877893769 \\ \mathrm{H} & -3.0052481616 & -3.0496005768 & 2.6134971457 \\ \mathrm{H} & -1.445739409 & -2.5024411846 & 3.173422646 \\ \mathrm{H} & -1.5505026998 & -3.5582421941 & 1.7660235024 \\ \mathrm{O} & -2.6942043087 & -1.9143863719 & 0.0898837543 \\ \mathrm{C} & -4.0148294849 & -2.4821187465 & 0.0557112627 \\ \mathrm{H} & -4.2392307644 & -2.638134368 & -0.9884400364 \\ \mathrm{H} & -4.7526198105 & -1.8141278144 & 0.4795578344 \\ \mathrm{H} & -4.0560511447 & -3.4322777047 & 0.5716453962\end{array}$

$H F=-1403.2906263$ 


\section{Revised carbon assignments: Exchange $\mathrm{C} 9$ and $\mathrm{C} 12$}

\begin{tabular}{rrrrrr}
\multicolumn{1}{c}{$\begin{array}{c}\text { Carbon } \\
\text { Number }\end{array}$} & mPW1PW91/ & (linear fit) & & \\
1 & & & & \\
2 & 18.6 & 18.9 & 14.6 & -4.0 & |difference \\
3 & 142.2 & 132.4 & 134.5 & -7.7 & 7.7 \\
4 & 26.1 & 27.7 & 23.9 & -2.2 & 2.2 \\
5 & 120.7 & 120.7 & 122.2 & 1.5 & 1.5 \\
6 & 75.8 & 84.1 & 83.5 & 7.7 & 7.7 \\
7 & 60.5 & 64.7 & 63.0 & 2.5 & 2.5 \\
8 & 202.9 & 200.1 & 206.1 & 3.2 & 3.2 \\
9 & 53.1 & 55.6 & 53.4 & 0.3 & 0.3 \\
10 & 40.4 & 40.3 & 37.2 & -3.2 & 3.2 \\
11 & 47.8 & 55.5 & 53.2 & 5.4 & 5.4 \\
12 & 71.5 & 73.5 & 72.3 & 0.8 & 0.8 \\
13 & 54.5 & 58.2 & 56.1 & 1.6 & 1.6 \\
14 & 72.7 & 76.1 & 75.0 & 2.3 & 2.3 \\
15 & 61 & 64.1 & 62.3 & 1.3 & 1.3 \\
16 & 53.2 & 53.5 & 51.1 & -2.1 & 2.1 \\
17 & 192.8 & 185.3 & 190.4 & -2.4 & 2.4 \\
18 & 132.5 & 128.1 & 130.0 & -2.5 & 2.5 \\
19 & 139.6 & 139.0 & 141.5 & 1.9 & 1.9 \\
20 & 40.9 & 57.5 & 55.4 & 14.5 & 14.5 \\
21 & 77.3 & 80.5 & 79.7 & 2.4 & 2.4 \\
22 & 26.6 & 27.1 & 23.2 & -3.4 & 3.4 \\
23 & 24.7 & 22.2 & 18.1 & -6.6 & 6.6 \\
& 49.1 & 47.8 & 45.1 & -4.0 & 4.0 \\
& & & & & 3.6 \\
& & & & average & \\
& & & & & 14.5
\end{tabular}

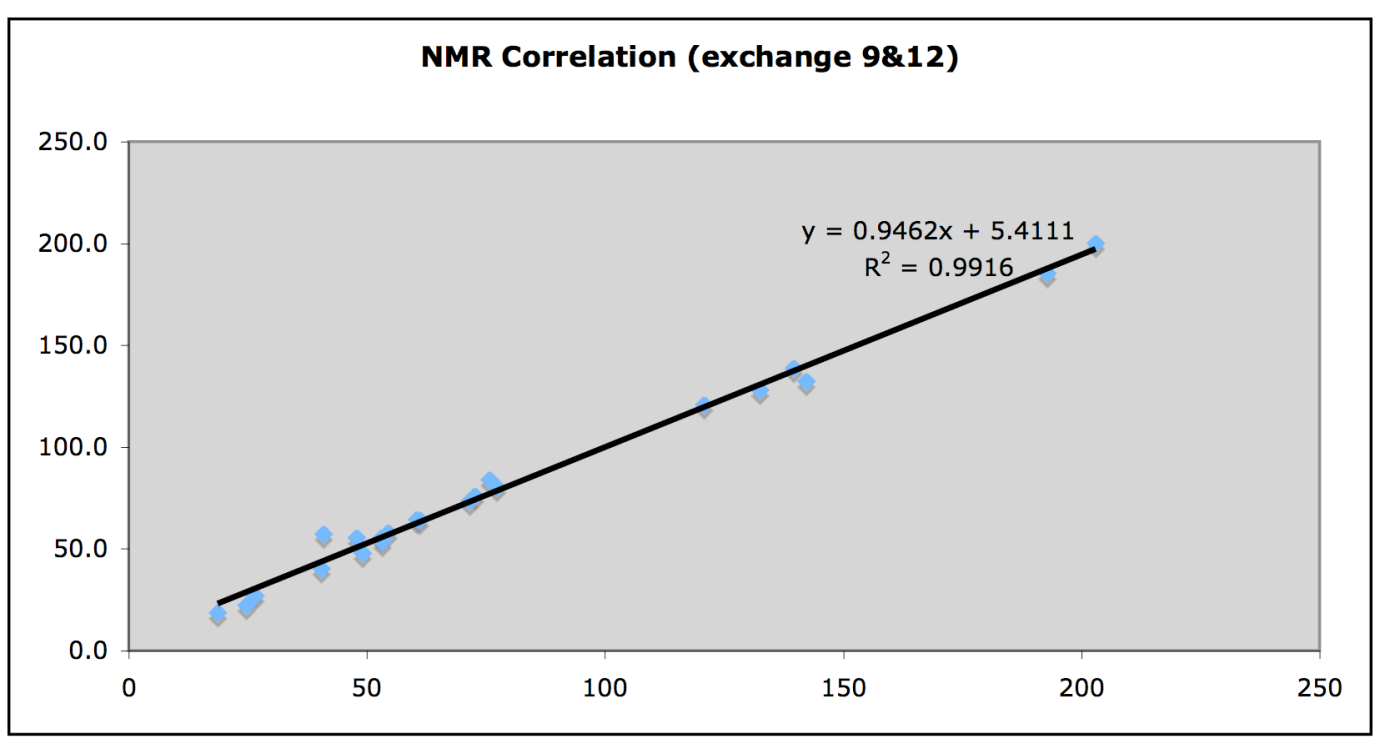




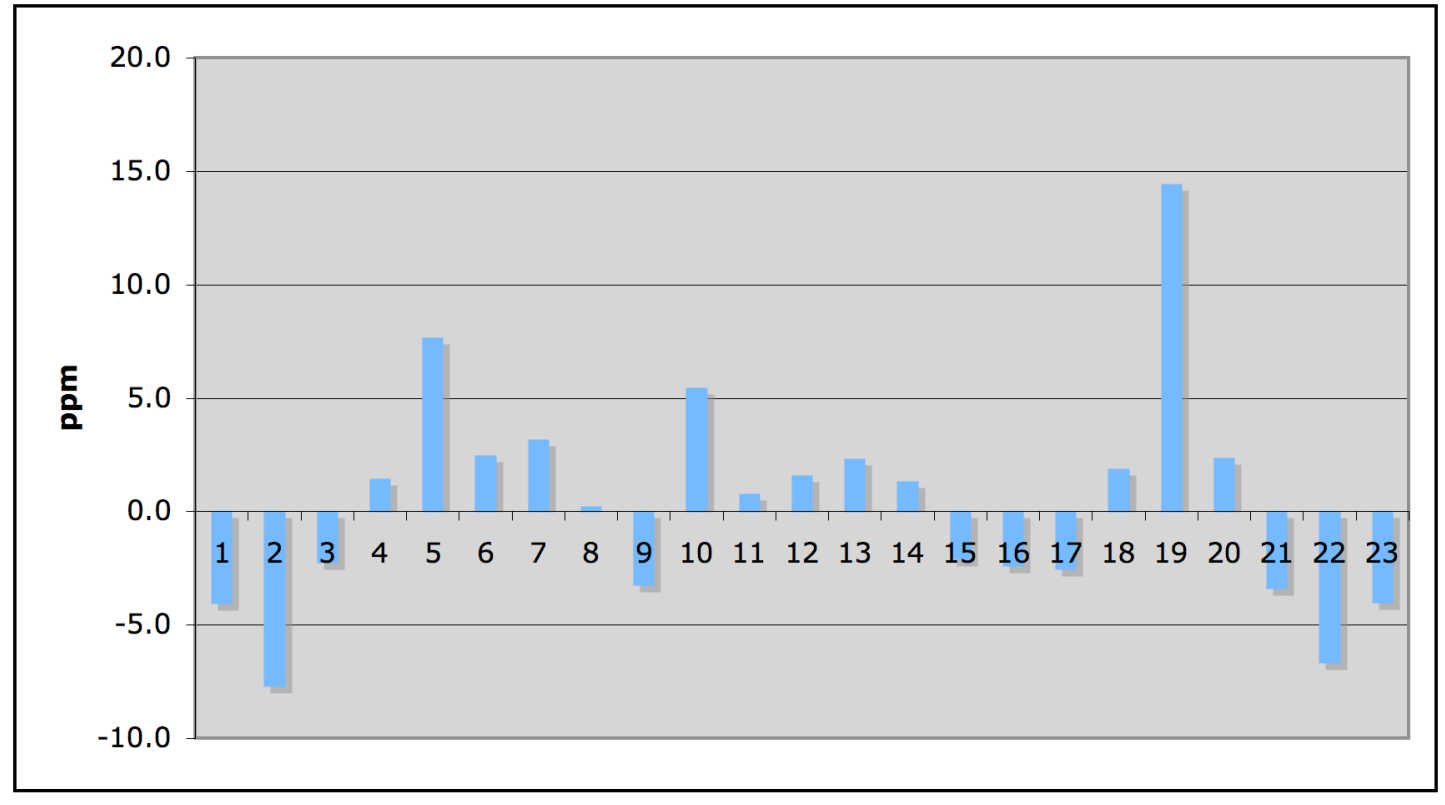


Compound 6-B (5-down \& 19-down) optimized at HF/3-21G

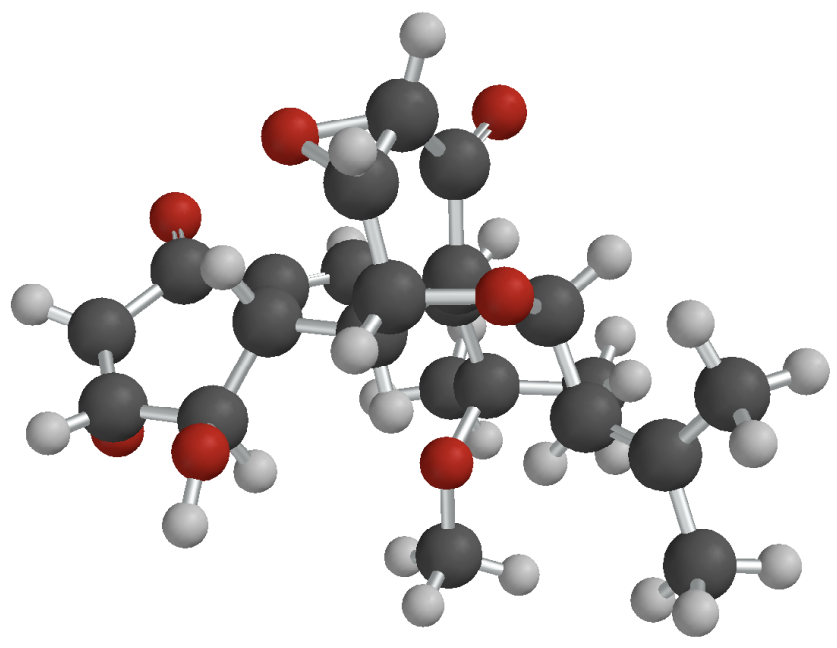

$\begin{array}{llll}\mathrm{C} & 1.114734084 & 1.2054772081 & 0.704340196 \\ \mathrm{C} & 1.1151737609 & 1.2033865351 & 3.2323836115 \\ \mathrm{C} & 3.3095419565 & 1.2059738527 & 1.88060719 \\ \mathrm{C} & 2.5868751668 & 1.4706180699 & 3.1393371271 \\ \mathrm{C} & 2.554119232 & 0.7142988635 & 0.6627949018 \\ \mathrm{C} & 0.4838168152 & 0.714438528 & 1.982766172 \\ \mathrm{H} & 4.2259687949 & 1.7295472801 & 1.6988472679 \\ \mathrm{H} & 2.5644357106 & -0.367195221 & 0.6546563299 \\ \mathrm{H} & 2.9847944241 & 2.141677214 & 3.8700053817 \\ \mathrm{C} & 0.3493439792 & 0.7221566353 & -0.5275375978 \\ \mathrm{H} & 0.8824556384 & -0.1275807602 & -0.9195925307 \\ \mathrm{C} & -1.0932677947 & 0.2284066538 & -0.3224288444 \\ \mathrm{C} & -0.5724975344 & -0.0679253984 & 2.0640352871 \\ \mathrm{H} & -0.9542058494 & -0.2880540159 & 3.041937651 \\ \mathrm{C} & -1.2703289376 & -0.7312100112 & 0.8901386228 \\ \mathrm{O} & 3.3620513204 & 0.2194993923 & 2.9662681772 \\ \mathrm{C} & -2.0794460238 & 1.3900949605 & -0.0418847419 \\ \mathrm{C} & 0.1510787096 & 1.6964451891 & -1.6883240435 \\ \mathrm{H} & 1.0585379279 & 1.859877804 & -2.2404838818 \\ \mathrm{C} & -0.5473677574 & 2.9739967612 & -1.296247555 \\ \mathrm{H} & -0.5114523982 & 3.7944949551 & -1.9813416775 \\ \mathrm{C} & -1.7448732166 & 2.7786329692 & -0.4804420914 \\ \mathrm{H} & -2.5792145288 & 3.4446353689 & -0.5202832772 \\ \mathrm{O} & 3.1242248653 & 1.2684665122 & -0.5425863559 \\ \mathrm{H} & 3.9257825917 & 0.7963292703 & -0.8034620498 \\ \mathrm{O} & -0.8448728635 & 1.0377212537 & -2.5313496289 \\ \mathrm{O} & -0.5246321731 & 3.3584079389 & 0.1172201267 \\ \mathrm{O} & 0.5301505998 & 1.329880596 & 4.2868743331 \\ \mathrm{O} & -3.1746008676 & 1.178270012 & 0.4332649469\end{array}$




$\begin{array}{llll}\mathrm{C} & -1.423765028 & -0.1213097477 & -1.8246700736 \\ \mathrm{H} & -2.4890462053 & -0.0862537291 & -1.9947111504 \\ \mathrm{C} & -0.8150679348 & -1.3639880696 & -2.4252677865 \\ \mathrm{H} & -0.1564605217 & -1.9209819484 & -1.798167927 \\ \mathrm{C} & -1.0278667431 & -1.7426859965 & -3.6722039213 \\ \mathrm{C} & -1.910907494 & -0.9951520662 & -4.6526980124 \\ \mathrm{H} & -1.4424744868 & -0.9809584147 & -5.6317810841 \\ \mathrm{H} & -2.0671194756 & 0.0270416204 & -4.345384556 \\ \mathrm{H} & -2.8700835985 & -1.496298811 & -4.7583660347 \\ \mathrm{C} & -0.3822713425 & -2.9946328717 & -4.2311125238 \\ \mathrm{H} & -1.1417611912 & -3.6777959206 & -4.6019767531 \\ \mathrm{H} & 0.2051881982 & -3.5090548357 & -3.4805961151 \\ \mathrm{H} & 0.2666655005 & -2.7444429248 & -5.065470771 \\ \mathrm{H} & 1.1354657481 & 2.2811275771 & 0.7317733594 \\ \mathrm{H} & -2.3278972604 & -0.7526653943 & 1.1119132447 \\ \mathrm{C} & -0.8268751281 & -2.2229341612 & 0.8085873305 \\ \mathrm{C} & -1.8700757738 & -3.0495908304 & 0.0327046668 \\ \mathrm{H} & -2.0879156272 & -2.6345174803 & -0.9360330429 \\ \mathrm{H} & -2.7847911549 & -3.0836607226 & 0.612092721 \\ \mathrm{H} & -1.5270942362 & -4.0684393648 & -0.1041865141 \\ \mathrm{C} & -0.7321491578 & -2.8297047001 & 2.2252636383 \\ \mathrm{H} & -0.6238950894 & -3.9060528005 & 2.1644899199 \\ \mathrm{H} & -1.6389079596 & -2.6225040412 & 2.7800094531 \\ \mathrm{H} & 0.1124475191 & -2.4270065407 & 2.7656815142 \\ \mathrm{O} & 0.4805345844 & -2.2258951213 & 0.1779294744 \\ \mathrm{C} & 1.2623927984 & -3.4371018945 & 0.1955628531 \\ \mathrm{H} & 2.1209388014 & -3.2427394069 & -0.4291449006 \\ \mathrm{H} & 0.716493934 & -4.2790065783 & -0.2099847318 \\ \mathrm{H} & 1.5984012521 & -3.6764697231 & 1.1943651356\end{array}$

$H F=-1403.276611$ 


\section{Revised carbon assignments: Exchange $\mathrm{C} 9$ and $\mathrm{C12}$} mPW1PW91/ (linear fit)

Carbon

Number
Chemical shift

$$
18.6
$$

142.2

26.1

120.7

75.8

60.5

202.9

53.1

40.4

47.8

71.5

$\mathbf{5 4 . 5}$

72.7

61

53.2

192.8

132.5

139.6

40.9

77.3

26.6

24.7

49.1
$6-31 G(d, p)$ 18.5 135.0

26.3

123.4

79.0

62.8

205.0

59.3

40.5

43.8

70.5

62.4

75.5

65.5

55.8

188.6

132.2

136.0

48.0

81.4

26.5

22.6

48.5
Corrected

16.2

135.4

24.2

123.5

78.1

61.5

207.0

57.9

38.7

42.1

69.4

61.1

74.5

64.3

54.4

190.2

132.5

136.4

46.4

80.5

24.4

20.4

46.9 difference

$-2.4$

$-6.8$

$-1.9$

2.8

2.3

1.0

4.1

4.8

$-1.7$

$-5.7$

$-2.1$

6.6

1.8

3.3

1.2

$-2.6$

0.0

$-3.2$

5.5

3.2

$-2.2$

$-4.3$

$-2.2$

average

$\max$ |difference|

2.4

6.8

1.9

2.8

2.3

1.0

4.1

4.8

1.7

5.7

2.1

6.6

1.8

3.3

1.2

2.6

0.0

3.2

5.5

3.2

2.2

4.3

2.2

3.1

6.8

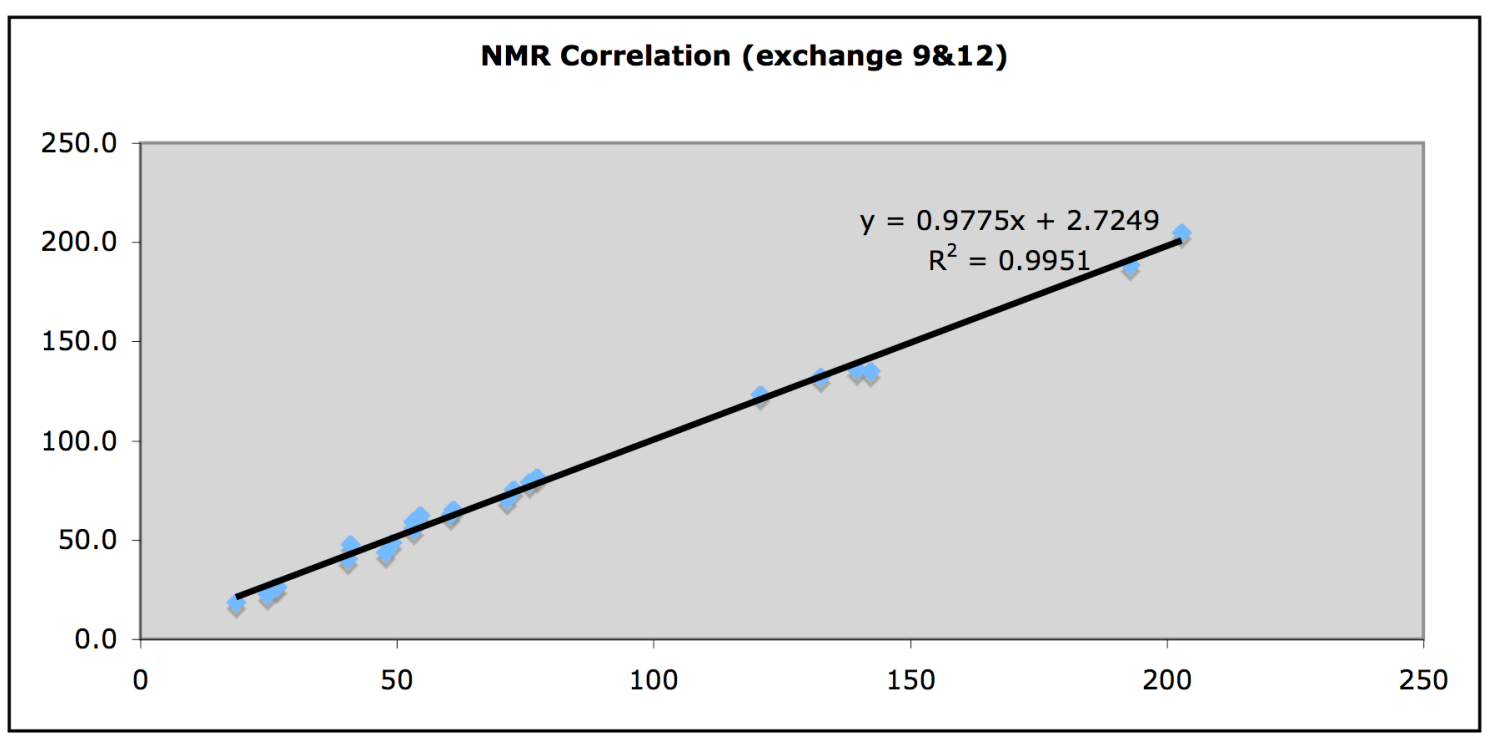




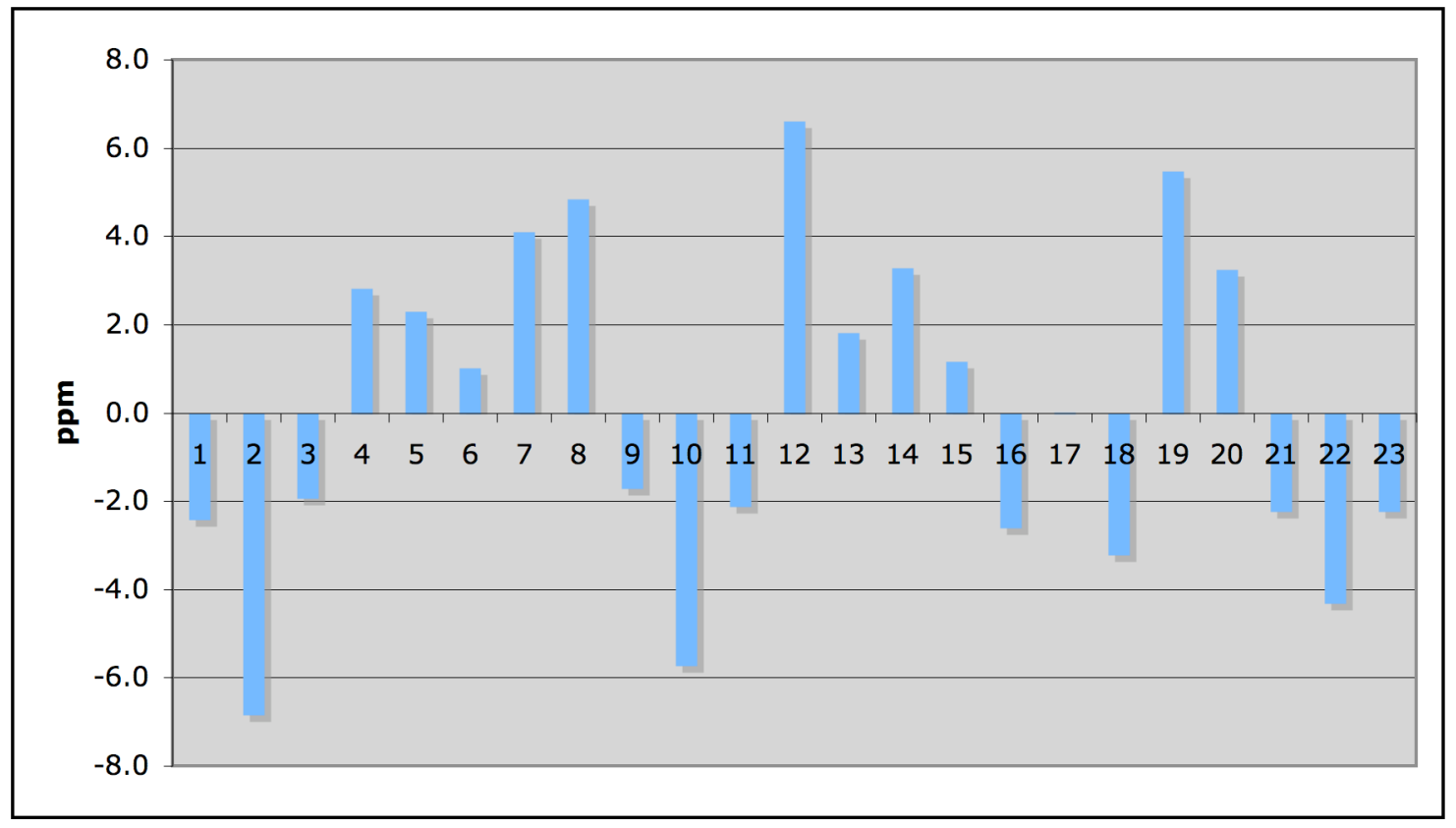


Compound 6-A (5-down \& 19-up) optimized at HF/3-21G

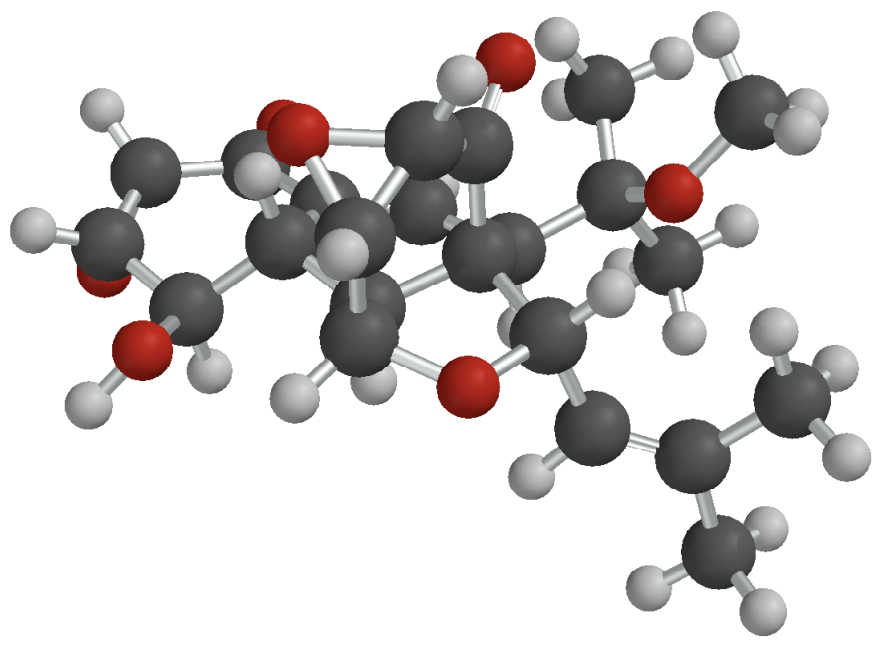

$\begin{array}{llll}\mathrm{C} & 1.9535479065 & 0.468275783 & 0.4868170761 \\ \mathrm{C} & 1.9524874283 & 0.4588679706 & 3.0678521814 \\ \mathrm{C} & 4.1201108068 & 0.4712725066 & 1.7107101613 \\ \mathrm{C} & 3.4116878925 & 0.7792171449 & 2.9590294704 \\ \mathrm{C} & 3.3419524719 & -0.1639306956 & 0.5796471953 \\ \mathrm{C} & 1.2313677137 & 0.1948656435 & 1.7944976589 \\ \mathrm{H} & 4.987303888 & 1.0385267883 & 1.4428131596 \\ \mathrm{H} & 3.2464169227 & -1.2217828923 & 0.79241741 \\ \mathrm{H} & 3.7712285761 & 1.5314504864 & 3.6284885958 \\ \mathrm{C} & 1.1496719929 & -0.1508816178 & -0.6516190543 \\ \mathrm{H} & 1.2711229724 & -1.2277742444 & -0.5870830625 \\ \mathrm{C} & -0.3716260291 & 0.1267718218 & -0.5664965379 \\ \mathrm{C} & -0.0185258183 & -0.2313177901 & 1.8759731693 \\ \mathrm{H} & -0.4024405255 & -0.4083933539 & 2.8605953214 \\ \mathrm{C} & -0.9286677682 & -0.5311490523 & 0.7057907095 \\ \mathrm{O} & 4.274539757 & -0.4196361122 & 2.872406557 \\ \mathrm{C} & -0.5639852834 & 1.6337828897 & -0.6245702846 \\ \mathrm{C} & 1.4371588421 & 0.2196023718 & -2.1050194258 \\ \mathrm{H} & 2.3697007353 & -0.1725948056 & -2.4572102755 \\ \mathrm{C} & 1.2537133486 & 1.6754408101 & -2.4241671762 \\ \mathrm{H} & 1.5067148653 & 1.980849033 & -3.417306871 \\ \mathrm{C} & 0.1601804842 & 2.3520915565 & -1.7314582804 \\ \mathrm{H} & -0.3683286127 & 3.1705515991 & -2.1716925339 \\ \mathrm{O} & 3.9965394165 & 0.0614096332 & -0.6837197251 \\ \mathrm{H} & 4.8076773225 & -0.4598129623 & -0.7542227707 \\ \mathrm{O} & 0.3420239431 & -0.4174244433 & -2.8105432111 \\ \mathrm{O} & 1.5729129842 & 2.6623678902 & -1.3873270033 \\ \mathrm{O} & 1.408388646 & 0.4360351144 & 4.1529434979 \\ \mathrm{O} & -1.2365602364 & 2.2922009317 & 0.1350879434\end{array}$




$\begin{array}{llll}\mathrm{C} & -0.8423895806 & -0.4918499772 & -1.937219836 \\ \mathrm{H} & -1.6266730278 & 0.0975157136 & -2.3701627911 \\ \mathrm{C} & -1.1849213448 & -1.9501944337 & -1.8835557154 \\ \mathrm{H} & -0.3449331105 & -2.5715857444 & -1.6302441242 \\ \mathrm{C} & -2.3279071295 & -2.5335452811 & -2.1920656554 \\ \mathrm{C} & -3.5954132833 & -1.8269396406 & -2.6188174781 \\ \mathrm{H} & -3.4652003132 & -0.7640543871 & -2.7291326479 \\ \mathrm{H} & -4.3721953083 & -1.9902200517 & -1.8786557872 \\ \mathrm{H} & -3.9417248214 & -2.2393301163 & -3.561584201 \\ \mathrm{C} & -2.4651854352 & -4.0442520494 & -2.1479914994 \\ \mathrm{H} & -3.2606412421 & -4.3319999896 & -1.4660870309 \\ \mathrm{H} & -1.5479334986 & -4.5216361409 & -1.8271172536 \\ \mathrm{H} & -2.7244638513 & -4.4252223433 & -3.131375284 \\ \mathrm{H} & 2.0761170518 & 1.5268667524 & 0.3193502797 \\ \mathrm{H} & -0.8442371727 & -1.5980825679 & 0.5310201425 \\ \mathrm{C} & -2.4537337148 & -0.2783677751 & 1.0147367592 \\ \mathrm{C} & -2.7133752439 & 0.7163308513 & 2.1612886728 \\ \mathrm{H} & -2.2314512147 & 1.6582055295 & 1.97359634 \\ \mathrm{H} & -2.3560121111 & 0.3040927902 & 3.0945232542 \\ \mathrm{H} & -3.7787898551 & 0.8714240951 & 2.2787194439 \\ \mathrm{C} & -3.1178286521 & -1.6172927622 & 1.3751857983 \\ \mathrm{H} & -4.172574908 & -1.4710419449 & 1.5801310077 \\ \mathrm{H} & -2.6579707103 & -2.0445466841 & 2.2595292974 \\ \mathrm{H} & -3.0162736956 & -2.3067405276 & 0.550040721 \\ \mathrm{O} & -3.0071123738 & 0.1527404355 & -0.25712966 \\ \mathrm{C} & -4.1578575521 & 1.0139469968 & -0.2772970351 \\ \mathrm{H} & -4.9914997013 & 0.5941332178 & 0.2724775788 \\ \mathrm{H} & -4.441310589 & 1.1034176733 & -1.3157292995 \\ \mathrm{H} & -3.9202574722 & 1.9917658518 & 0.1126489468\end{array}$

$H F=-1403.288462$ 


\section{Revised carbon assignments: Exchange $\mathrm{C} 9$ and $\mathrm{C12}$}

\begin{tabular}{|c|c|c|c|c|c|}
\hline \multicolumn{6}{|l|}{ Carbon } \\
\hline $\begin{array}{l}\text { Carbon } \\
\text { Number }\end{array}$ & Chemical shift & $6-31 G(d, p)$ & Corrected & difference & |difference| \\
\hline 1 & 18.6 & 20.4 & 17.0 & -1.6 & 1.6 \\
\hline 2 & 142.2 & 133.0 & 135.3 & -6.9 & 6.9 \\
\hline 3 & 26.1 & 26.5 & 23.4 & -2.7 & 2.7 \\
\hline 4 & 120.7 & 120.5 & 122.2 & 1.5 & 1.5 \\
\hline 5 & 75.8 & 78.9 & 78.5 & 2.7 & 2.7 \\
\hline 6 & 60.5 & 62.5 & 61.3 & 0.8 & 0.8 \\
\hline 7 & 202.9 & 200.8 & 206.6 & 3.7 & 3.7 \\
\hline 8 & 53.1 & 54.6 & 53.0 & -0.1 & 0.1 \\
\hline 9 & 40.4 & 39.1 & 36.7 & -3.7 & 3.7 \\
\hline 10 & 47.8 & 51.2 & 49.4 & 1.6 & 1.6 \\
\hline 11 & 71.5 & 73.6 & 72.9 & 1.4 & 1.4 \\
\hline 12 & 54.5 & 59.6 & 58.2 & 3.7 & 3.7 \\
\hline 13 & 72.7 & 76.6 & 76.1 & 3.4 & 3.4 \\
\hline 14 & 61 & 63.5 & 62.3 & 1.3 & 1.3 \\
\hline 15 & 53.2 & 52.9 & 51.2 & -2.0 & 2.0 \\
\hline 16 & 192.8 & 184.8 & 189.7 & -3.1 & 3.1 \\
\hline 17 & 132.5 & 127.5 & 129.5 & -3.0 & 3.0 \\
\hline 18 & 139.6 & 140.6 & 143.3 & 3.7 & 3.7 \\
\hline 19 & 40.9 & 53.0 & 51.3 & 10.4 & 10.4 \\
\hline 20 & 77.3 & 79.3 & 78.9 & 1.6 & 1.6 \\
\hline 21 & 26.6 & 29.1 & 26.2 & -0.4 & 0.4 \\
\hline 22 & 24.7 & 22.2 & 18.9 & -5.8 & 5.8 \\
\hline 23 & 49.1 & 49.8 & 47.9 & -1.2 & 1.2 \\
\hline & & & & average & 2.9 \\
\hline & & & & $\max$ & 10.4 \\
\hline
\end{tabular}

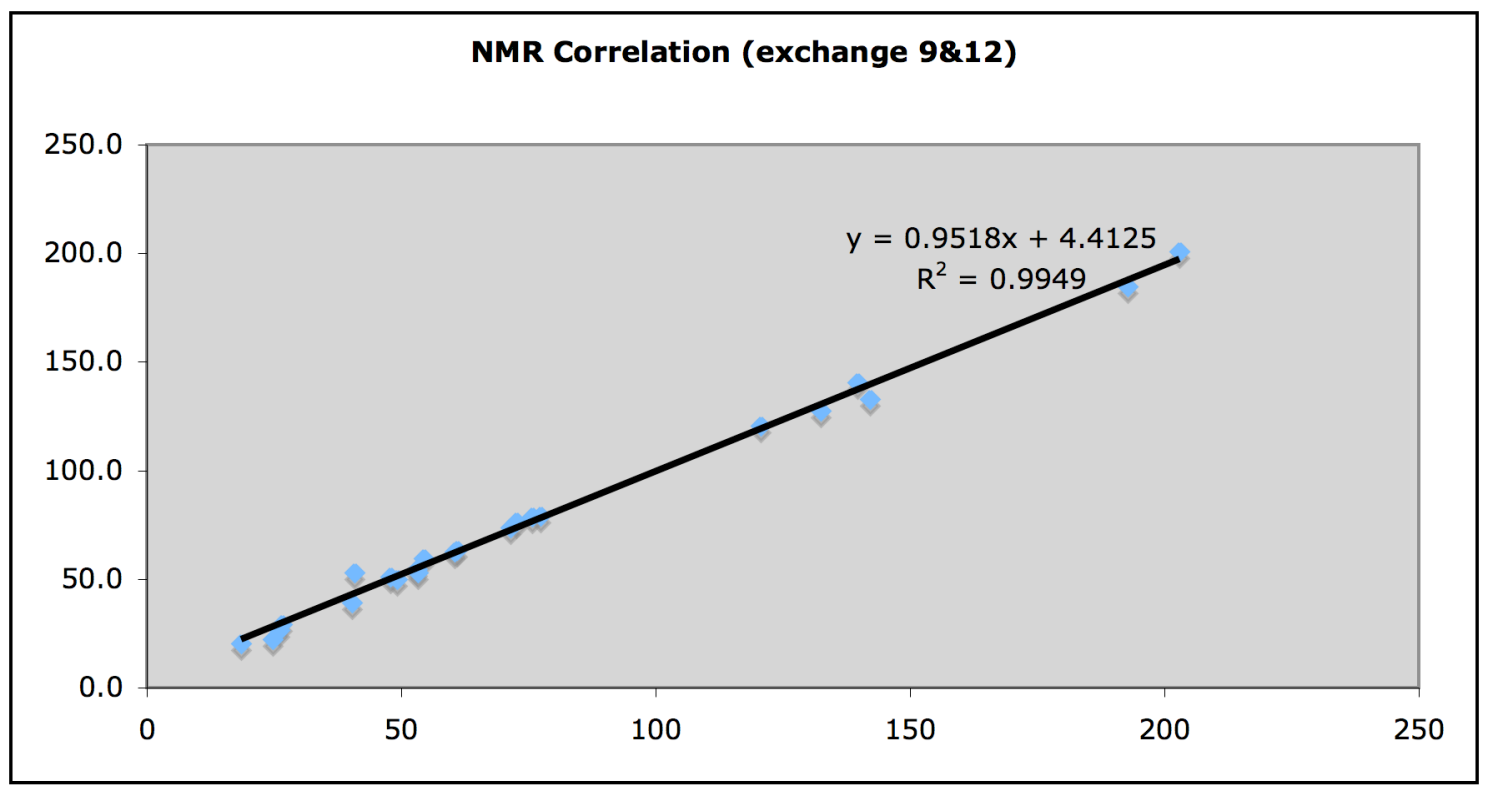




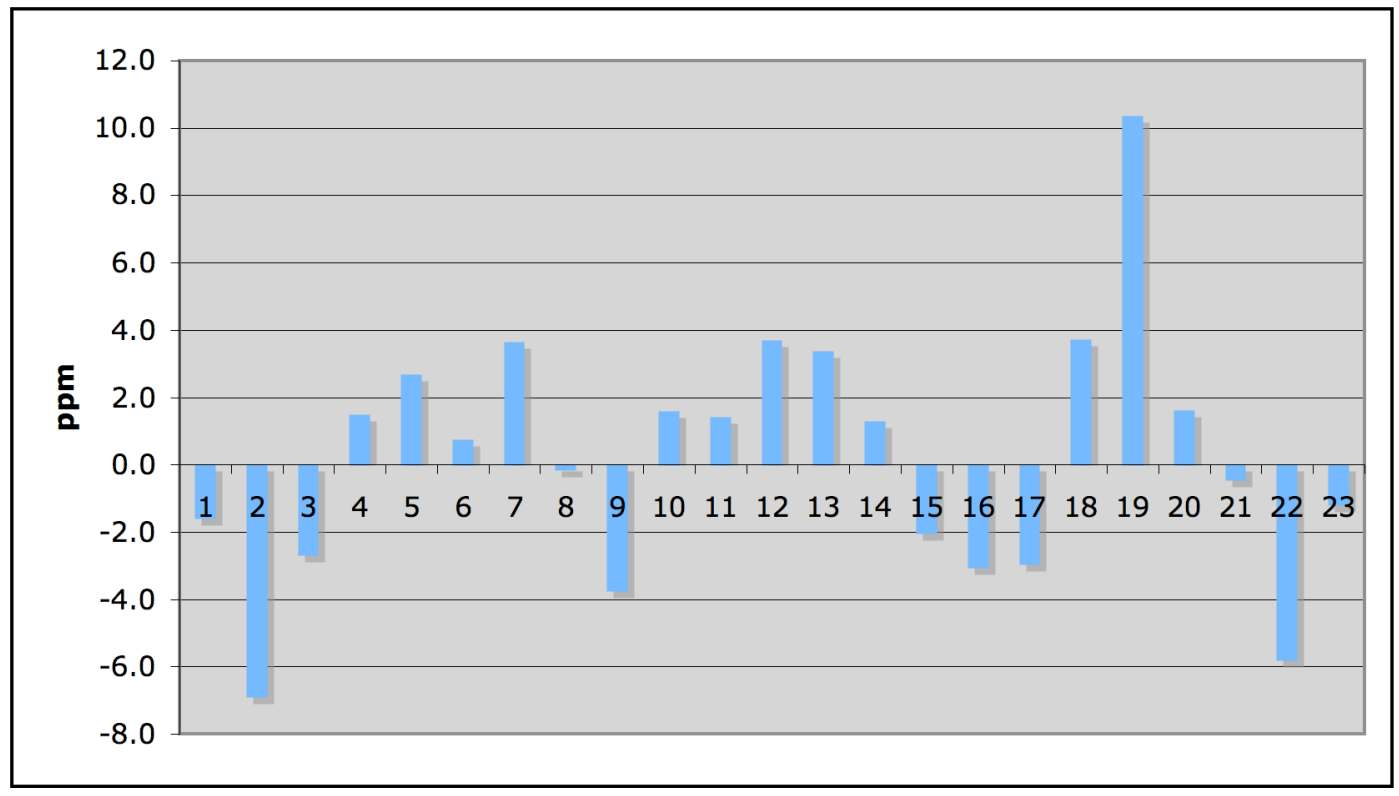

